\title{
Gluon self-energy in the Coulomb and temporal axial gauges via the pinch technique
}

\author{
Massimo Passera* and Ken Sasaki ${ }^{\dagger}$ \\ Department of Physics, New York University, 4 Washington Place, New York, New York 10003
}

(Received 5 June 1996)

\begin{abstract}
The $S$-matrix pinch technique is used to derive an effective gluon self-energy to one-loop order, when the theory is quantized in the Coulomb gauge $(\mathrm{CG})$ and in the temporal axial gauge (TAG). When the pinch contributions are added, the gluon self-energies calculated in the CG and TAG turn out to be identical and coincide with the result previously obtained with covariant gauges. The issue of gauge independence of several quantities in hot QCD is discussed from the pinch technique point of view. It is also pointed out that the spurious singularities which appear in TAG calculations cancel out once the pinch contributions are combined. [S0556-2821(96)05521-X]

PACS number(s): 12.38.Bx, 11.10.Wx, 11.15.Bt
\end{abstract}

\section{INTRODUCTION}

The $S$-matrix pinch technique (PT) is an algorithm which enables us to construct gauge-independent (GI) modified offshell $n$-point functions through the rearrangement of Feynman graphs contributing to certain physical $S$-matrix elements. First introduced by Cornwall [1] some time ago to form the new GI-QCD proper vertices and propagators for the Schwinger-Dyson equations, the PT was used to obtain the one-loop GI effective gluon self-energy and vertices in QCD [2,3]. It has then been extensively applied to the standard model [4]. Recently the PT was applied also to QCD at high temperature to calculate the gap equation for the magnetic mass [5] and to obtain the GI thermal $\beta$ function $[6,7]$.

Indeed, the PT algorithm has scored a success in its applications to various fields. However, we can hardly say that it was fully understood and well established. In particular, since in the $S$-matrix PT the effective amplitudes are obtained through the rearrangement of Feynman graphs, their uniqueness is at stake. One may argue that arbitrary pieces can always be moved around by hand from the vertex or box diagrams, as long as one does not alter the unique $S$-matrix element. On the other hand, the $S$-matrix PT algorithm is expected to give rise to the same answers, even when one may choose an $S$-matrix element for a different process or start calculations with different gauge-fixing choices. Unfortunately, there exists so far no general proof on this point, and, therefore, we may have to examine individual cases to convince ourselves of the validity of the PT algorithm. The process independence of the PT has been recently proved [8] via explicit one-loop calculations. The independence of the gauge-fixing choices has been shown for the case of the effective gluon self-energy at one-loop order in the covariant gauge [2], the background field gauge $[9,10]$ and one of the noncovariant gauges, namely, the light-cone gauge [1]. How-

\footnotetext{
*Electronic address: passera@mafalda.physics.nyu.edu

${ }^{\dagger}$ Permanent address: Dept. of Physics, Yokohama National University, Yokohama 240, Japan. Electronic address: sasaki@mafalda.physics.nyu.edu or sasaki@ed.ynu.ac.jp
}

ever, the PT calculations have not been carried out in the other interesting noncovariant gauges up to the present.

Noncovariant gauges such as the Coulomb gauge (CG) and the axial gauges have long been used, both for theoretical analyses and for various numerical calculations in gauge theories [11]. These gauges are sometimes called "physical", gauges since in these gauges there is a close correspondence between independent fields and "physical" degrees of freedom. In particular, the $\mathrm{CG}$ and the temporal axial gauge (TAG) have been often chosen for the perturbative calculations of QCD at finite temperature [12-14]. The reasons for these gauges being used are, for the $\mathrm{CG}$, that it is a natural gauge choice for the study of interactions between charges and, for the TAG, that for a thermal system the rest frame of the heat bath singles out the four-vector $n^{\mu}=(1.0 .0,0)$ [15].

The gluon self-energy is a gauge-dependent quantity. Its one-loop expression in the $\mathrm{CG}$ differs from the one in the TAG. And the transversality relation is satisfied by the oneloop gluon self-energy calculated in the TAG but not by the one in the CG. However, the hard thermal loop $\delta \Pi_{\mu \nu}$ in the gluon self-energy is gauge independent, which means that $\delta \Pi_{\mu \nu}$ 's calculated in the CG and in the TAG are the same. The electric mass $m_{\mathrm{el}}$, relevant for electric screening, and the "effective gluon mass" $m_{G}$ in hot QCD are gauge independent quantities and they can be obtained from the one-loop gluon self-energy calculated in any gauge choice. Meanwhile it is well known that in TAG calculations there appear spurious singularities which are due to the unphysical poles of $(k \cdot n)^{-\lambda}, \lambda=1,2$ in the TAG gluon propagator $[11,16]$. Several methods have been proposed to circumvent these singularities, and most noticeable are the principal-value prescription [17], the $n_{\mu}^{*}$ prescription [18], and the $\alpha$ prescription [19].

In this paper we apply the $S$-matrix PT and calculate an effective gluon self-energy to one-loop order in the $\mathrm{CG}$ and the TAG. The one-loop gluon self-energies both in the $\mathrm{CG}$ and the TAG have very complicated expressions. Even in these gauges we find that once the pinch contributions are added, we indeed obtain the same result for the effective gluon self-energy as the one derived before in different gauge choices. This gives another support for the usefulness of the $S$-matrix PT. We can also argue why the transversality 


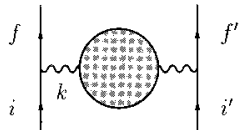

(a)

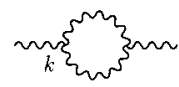

(b)

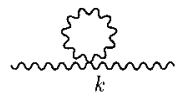

(c)

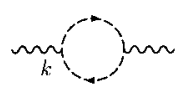

(d)
FIG. 1. (a) The gluon self-energy diagrams for the quark-quark scattering. (b) The gluon self-energy diagram with three-gluon interactions. (c) The tadpole diagram for the gluon self-energy. (d) The ghost diagram for the gluon self-energy.

relation holds for the gluon self-energy calculated in the TAG, but not for the one in the CG, from the analysis of the structure of the pinch contributions. Moreover, we can explain why the thermal loops, the electric mass $m_{\mathrm{el}}$, and the effective gluon mass $m_{G}$ in hot QCD are gauge independent from a simple inspection of the pinch contributions. Concerning the spurious singularities which appear in the gluon self-energy in the TAG, we point out that these singularities also appear in the pinch contributions and they exactly cancel against the counterparts in the gluon self-energy. To show explicitly how these cancellations occur, we calculate in the TAG the one-loop gauge-independent thermal $\beta$ function $\beta_{T}$ in hot QCD.

The paper is organized as follows. In the next section, we develop the general prescription necessary for extracting the pinch contributions to the gluon self-energy from the oneloop quark-quark scattering amplitude. To establish our notation and to illustrate how to use the prescription developed in the previous section, we briefly review, in Sec. III, the derivation of the pinch contribution to the gluon self-energy in the Feynman gauge (FG). In Sec. IV we calculate both the gluon self-energy and the pinch contribution in one-loop order in the $\mathrm{CG}$ with an arbitrary gauge parameter $\xi_{C}$, and show that when combined they give the same expression for the effective gluon self-energy as the one obtained before in different gauge choices. In Sec. V the similar calculations are performed in the TAG with an arbitrary gauge parameter $\xi_{A}$. Also we calculate the thermal $\beta$ function $\beta_{T}$ at one-loop order in the TAG and show how the spurious singularities appearing in the TAG gluon self-energy cancel against the counterparts in the pinch contribution. Section VI is devoted to summary and discussion. In addition, we present three
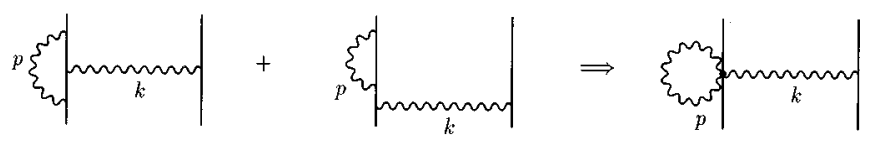

(a)

(b)

FIG. 2. (a) The vertex diagrams of the first kind for the quarkquark scattering. (b) Their pinch contribution.

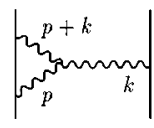

(a)

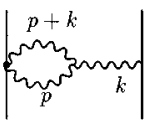

(b)
FIG. 3. (a) The vertex diagram of the second kind for the quarkquark scattering. (b) Its pinch contribution.

Appendices. In Appendix A we first give the one-loop pinch contributions to the gluon self-energy in the $\mathrm{CG}$ with $\xi_{C} \neq 0$ from the vertex diagrams of the first and second kind and from box diagrams, separately. Then we give the expression of the pinch contribution rewritten in terms of different tensor bases. In Appendix B, we give the similar expressions calculated in the TAG with $\xi_{A} \neq 0$. In Appendix $\mathrm{C}$ we list the formulas for thermal one-loop integrals necessary for calculating $\beta_{T}$ in the TAG in Sec. V.

\section{PINCH TECHNIQUE}

In this section we explain how to obtain the one-loop pinch contributions to the gluon self-energy. Let us consider the $S$-matrix element $T$ for the elastic quark-quark scattering at one-loop order in the Minkowski space, assuming that quarks have the same mass $m$. Throughout this paper we use the metric $(+,-,-,-)$. In addition to the self-energy diagram in Fig. 1(a), the vertex diagrams of the first and second kind and the box diagrams contribute to $T$. They are shown in Figs. 2(a), 3(a), and 4(a), respectively. These contributions are, in general, gauge dependent, while the sum is gauge independent. Then we single out the "pinch parts" of the vertex and box diagrams, which are depicted in Figs. 2(b), 3(b), and 4(b). They emerge when a $\gamma^{\mu}$ matrix on the quark line is contracted with a four-momentum $k_{\mu}$ offered by a gluon propagator or a bare three-gluon vertex. Such a term triggers an elementary Ward identity of the form

$$
k=(\not p+k-m)-(\not p-m) .
$$

The first term removes (pinches out) the internal quark propagator, whereas the second term vanishes on shell, or vice versa. This procedure leads to contributions to $T$ with one or two less quark propagators and, hence, we will call these contributions $T_{P}$, "pinch parts" of $T$.

Next we extract from $T_{P}$ the pinch contributions to the gluon self-energy $\Pi^{\mu \nu}$. First note that the contribution of the gluon self-energy diagram to $T$ is written in the form [see Fig. 1(a)]
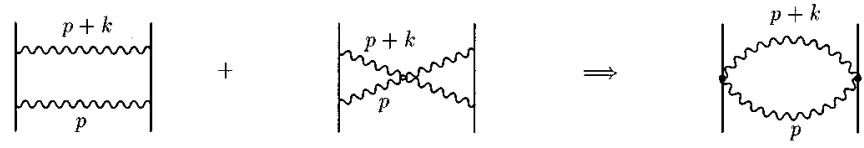

(a)

(b)

FIG. 4. (a) The box diagrams for the quark-quark scattering. (b) Their pinch contribution. 


$$
T^{(\mathrm{SE})}=\left[T^{a} \gamma^{\alpha}\right] D_{\alpha \mu}(k) \Pi^{\mu \nu} D_{\nu \beta}(k)\left[T^{a} \gamma^{\beta}\right],
$$

where $D(k)$ is a gluon propagator, $T^{a}$ is a representation matrix of $\operatorname{SU}(N)$, and $\gamma^{\alpha}$ and $\gamma^{\beta}$ are $\gamma$ matrices on the external quark lines. The pinch contribution $\Pi_{P}^{\mu \nu}$ to $T_{P}$ should have the same form. Thus we must take away $\left[T^{a} \gamma^{\alpha}\right] D_{\alpha \mu}(k)$ and $D^{\nu \beta}(k)\left[T^{a} \gamma^{\beta}\right]$ from $T_{P}$. For that purpose we use the following identity satisfied by the gluon propagator and its inverse:

$$
\begin{aligned}
g_{\alpha} \beta & =D_{\alpha \mu}(k)\left[D^{-1}\right]^{\mu \beta}(k)=D_{\alpha \mu}(k)\left[-k^{2} d^{\mu \beta}\right]+k_{\alpha} \text { term } \\
& =D_{\alpha \mu}^{-1}(k) D^{\mu \beta}(k)=\left[-k^{2} d_{\alpha \mu}\right] D^{\mu \beta}(k)+k_{\beta} \text { term, }
\end{aligned}
$$

where

$$
d^{\mu \nu}=g^{\mu \nu}-\frac{k^{\mu} k^{\nu}}{k^{2}} .
$$

The $k_{\alpha}$ and $k_{\beta}$ terms give null results when they are contracted with $\gamma_{\alpha}$ and $\gamma_{\beta}$, respectively, of the external quark lines.

The pinch part of the one-loop vertex diagrams of the first kind depicted in Fig. 2(b) plus their mirror graphs has a form

$$
T_{P}^{\left(V_{1}\right)}=\mathcal{A}\left[T^{a} \gamma^{\alpha}\right] D_{\alpha \beta}(k)\left[T^{a} \gamma^{\beta}\right],
$$

where $\mathcal{A}$ (also $\mathcal{B}_{0}, \mathcal{B}_{i j}, \mathcal{C}_{0}$, and $\mathcal{C}_{i j}$ in the equations below) contains a loop integral. Using Eq. (3) we find

$$
\gamma^{\alpha} D_{\alpha \beta}(k) \gamma^{\beta}=\gamma^{\alpha} D_{\alpha \mu}(k)\left[-k^{2} d^{\mu \nu}\right] D_{\nu \beta}(k) \gamma^{\beta} \text {. }
$$

Thus the contributions to $\Pi^{\mu \nu}$ from the vertex diagrams of the first kind are written as

$$
\Pi_{P}^{\mu \nu\left(V_{1}\right)}=\left[-k^{2} d^{\mu \nu}\right] \mathcal{A} .
$$

The pinch part of the one-loop vertex diagrams of the second kind depicted in Fig. 3(b) has a form

$$
T_{P}^{\left(V_{2}\right)}=\left[T^{a}\left\{\left[\gamma^{\kappa}\right] \mathcal{B}_{0}+\sum_{i, j} \mathcal{B}_{i j}\left[p_{i}\right] p_{j}^{\kappa}\right\}\right] D_{\kappa \beta}(k)\left[T^{a} \gamma^{\beta}\right],
$$

where $p_{i}$ and $p_{j}$ are four-momenta appearing in the diagrams. By redefinition of the loop-integral momentum we can choose $p_{i}, p_{j}=p$ or $n$ in the cases of the CG and the TAG where $p$ is the loop-integral momentum and $n$ is a unit vector $n^{\mu}=(1,0,0,0)$ appearing in the CG and TAG gluon propagators. Using Eq. (6) and

$$
\left[p_{i}\right] p_{j}^{\kappa} D_{\kappa \beta}(k)=\left[\gamma^{\alpha}\right] D_{\alpha \mu}(k)\left[-k^{2} d^{\mu \lambda}\right] p_{i \lambda} p_{j}^{\nu} D_{\nu \beta}(k),
$$

we obtain for the contributions to $\Pi^{\mu \nu}$ from the vertex diagrams of the second kind

$$
\Pi_{P}^{\mu \nu\left(V_{2}\right)}=\left[-k^{2} d^{\mu \nu}\right] \mathcal{B}_{0}+\left[-k^{2} d^{\mu \lambda}\right] \sum_{i, j} \mathcal{B}_{i j} p_{i \lambda} p_{j}^{\nu}+(\mu \leftrightarrow \nu),
$$

where $(\mu \leftrightarrow \nu)$ terms are the contributions from mirror diagrams. A further simplification can be made by using a formula

$$
k^{2} p_{j}^{\nu}=k^{2} d^{\nu \tau} p_{j \tau}+k^{\nu}\left(k p_{j}\right)
$$

The pinch part of the one-loop box diagrams depicted in Fig. 4(b) has a form

$$
T_{P}^{(\text {box })}=\left[T^{a}\right]\left\{\left[\gamma^{\alpha}\right]\left[\gamma_{\alpha}\right] \mathcal{C}_{0}+\sum_{i, j} \mathcal{C}_{i j}\left[p_{i}\right]\left[\not p_{j}\right]\right\}\left[T^{a}\right] .
$$

Again from Eq. (3) we see that $\left[\gamma^{\alpha}\right]\left[\gamma_{\alpha}\right]$ and $\left[p_{i}\right]\left[p_{j}\right]$ are rewritten as

$$
\begin{gathered}
{\left[\gamma^{\alpha}\right]\left[\gamma_{\alpha}\right]=\left[\gamma^{\alpha}\right] D_{\alpha \mu}(k)\left[k^{4} d^{\mu \nu}\right] D_{\nu \beta}(k)\left[\gamma_{\beta}\right]} \\
{\left[p_{i}\right]\left[p_{j}\right]=\left[\gamma^{\alpha}\right] D_{\alpha \mu}(k)\left[k^{4} d^{\mu \lambda} d^{\nu \tau} p_{i \lambda} p_{j \tau}\right] D_{\nu \beta}(k)\left[\gamma_{\beta}\right]}
\end{gathered}
$$

and thus we obtain for the contributions to $\Pi^{\mu \nu}$ from the box diagrams

$$
\Pi_{P}^{\mu \nu(\text { box })}=\left[k^{4} d^{\mu \nu}\right] \mathcal{C}_{0}+\left[k^{4} d^{\mu \lambda} d^{\nu \tau}\right] \sum_{i, j} \mathcal{C}_{i j} p_{i \lambda} p_{j \tau}
$$

It is observed that the prescription developed here is general and can be applied to the calculation of the one-loop pinch contributions in any gauge.

\section{PT GLUON SELF-ENERGY IN THE FEYNMAN GAUGE}

In order to establish our notation, in this section we briefly review the derivation of the effective gluon selfenergy in the Feynman gauge (FG) (the covariant gauge with $\xi=1)$. In the following we discuss the gluon self-energy both at $T=0$ and at finite temperature. In both cases we use the same notation $\int d p$ for the loop integral. At $T=0$ the loop integral should read as

$$
\int d p=-i \mu^{4-D} \int \frac{d^{D} p}{(2 \pi)^{D}}
$$

where $\mu$ is the ' $t$ Hooft mass scale, while at finite temperature we use the imaginary time formalism of thermal field theory, and the loop integral should read as

$$
\int d p=\int \frac{d^{3} p}{8 \pi^{3}} T \sum_{n} \quad \text { (imaginary time formalism), }
$$

where the summation goes over the integer $n$ in $p_{0}=2 \pi i n T$.

In the FG the gluon propagator, $i D_{a b(\mathrm{FG})}^{\mu \nu}=i \delta_{a b} D_{(\mathrm{FG})}^{\mu \nu}$, has a very simple form

$$
D_{(\mathrm{FG})}^{\mu \nu}(k)=\frac{-1}{k^{2}} g^{\mu \nu}
$$


and the three-gluon vertex is expressed as

$$
\Gamma_{\lambda \mu \nu}^{a b c}(p, k, q)=-g f^{a b c}\left[\Gamma_{\lambda \mu \nu}^{P}(p, k, q)+\Gamma_{\lambda \mu \nu}^{F}(p, k, q)\right],
$$

where

$$
\begin{gathered}
\Gamma_{\lambda \mu \nu}^{P}(p, k, q)=p_{\lambda} g_{\mu \nu}-q_{\nu} g_{\lambda \mu}, \\
\Gamma_{\lambda \mu \nu}^{F}(p, k, q)=2 k_{\lambda} g_{\mu \nu}-2 k_{\nu} g_{\lambda \mu}-(2 p+k)_{\mu} g_{\lambda \nu},
\end{gathered}
$$

and $f^{a b c}$ are the structure constants of the group $\mathrm{SU}(N)$. In the vertex each momentum flows inward and, thus, $p+k+q$ $=0$. The expression of the one-loop gluon self-energy in the FG is well known:

$$
\begin{aligned}
\Pi_{(\mathrm{FG})}^{\mu \nu}(k)= & N g^{2} \int d p \frac{1}{p^{2} q^{2}}\left[2 p^{\mu} p^{\nu}+2 q^{\mu} q^{\nu}\right. \\
& \left.-\left(p^{2}+q^{2}\right) g^{\mu \nu}-k^{\mu} k^{\nu}+2 k^{2} d^{\mu \nu}\right] .
\end{aligned}
$$

The one-loop pinch contribution to the gluon self-energy in the FG is calculated as follows. We consider the $S$-matrix element $T$ for the quark-quark scattering at one-loop order. Since the gluon propagator in the FG does not have a longitudinal $k^{\mu} k^{\nu}$ term, the pinch contribution to $T$ only comes from the vertex diagram of the second kind with the threegluon vertex of the type $\Gamma^{P}$ (and its mirror graph) [2], and is given by

$$
T_{P(\mathrm{FG})}^{V_{2}}=-2 N g^{2}\left[T^{a} \gamma^{\alpha}\right] \int d p \frac{1}{p^{2} q^{2}} D_{\alpha \beta}(k)\left[T^{a} \gamma^{\beta}\right] .
$$

The inverse of the gluon propagator is

$$
\left[D_{(\mathrm{FG})}^{-1}\right]^{\mu \nu}(k)=-k^{2} g^{\mu \nu},
$$

and thus $D_{(\mathrm{FG})}$ and its inverse satisfy the identities in Eq. (3). We can then apply the formulas Eqs. (8) and (10) to $T_{P(\mathrm{FG})}^{V_{2}}$ obtaining the FG pinch contribution to the gluon self-energy:

$$
\Pi_{P(\mathrm{FG})}^{\mu \nu}(k)=2 N g^{2} k^{2} d^{\mu \nu} \int d p \frac{1}{p^{2} q^{2}} .
$$

The sum of $\Pi_{(\mathrm{FG})}^{\mu \nu}$ and $\Pi_{P(\mathrm{FG})}^{\mu \nu}$ is given by

$$
\begin{aligned}
\hat{\Pi}^{\mu \nu}(k)= & N g^{2} \int d p \frac{1}{p^{2} q^{2}}\left[2 p^{\mu} p^{\nu}+2 q^{\mu} q^{\nu}\right. \\
& \left.-\left(p^{2}+q^{2}\right) g^{\mu \nu}-k^{\mu} k^{\nu}+4 k^{2} d^{\mu \nu}\right] .
\end{aligned}
$$

This is the effective gluon self-energy obtained before in the PT framework $[1,2,10]$. It is noted that $\hat{\Pi}^{\mu \nu}(k)$ can also be derived without using PT but by the background field method with a special value of the gauge parameter $\xi_{Q}=1$ [9].

The effective gluon self-energy $\hat{\Pi}^{\mu \nu}(k)$ has the following features.

(i) It satisfies the transversality relation. Indeed using $k+p+q=0$ we find
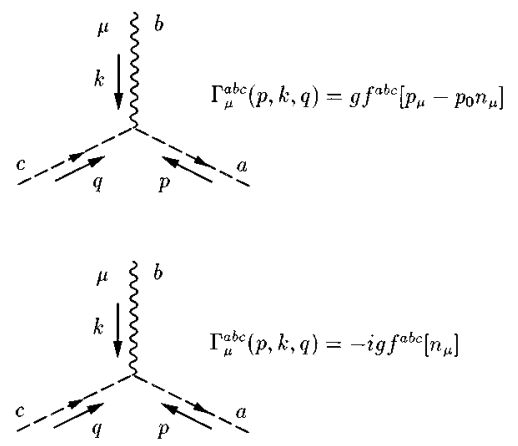

FIG. 5. (a) The ghost-gluon vertex in Coulomb gauge and (b) in temporal axial gauge.

$$
\hat{\Pi}^{\mu \nu}(k) k_{\nu}=2 N g^{2} \int d p\left\{\frac{p^{\mu}}{p^{2}}+\frac{q^{\mu}}{q^{2}}\right\}=0 .
$$

(ii) As was shown explicitly at one-loop level [2], the PT modified gluon three-point function $g f^{a b c} \hat{\Gamma}_{\mu \nu \alpha}$ and $\hat{\Pi}^{\mu \nu}(k)$ satisfy the following tree-level Ward-Takahashi identity

$$
p^{\mu} \hat{\Gamma}_{\mu \nu \alpha}(p, q, r)=-\hat{\Pi}_{\nu \alpha}(q)+\hat{\Pi}_{\nu \alpha}(r) .
$$

This implies that the wave function renormalization for the PT modified gluon self-energy $\hat{\Pi}_{\mu \nu}$ contains the running of the QCD couplings. Indeed, at zero temperature, after integration and renormalization it is rewritten as

$$
\hat{\Pi}^{\mu \nu}(k)=g^{2}\left(g^{\mu \nu} k^{2}-k^{\mu} k^{\nu}\right)\left(b \ln \frac{k^{2}}{\mu^{2}}+\text { const }\right),
$$

where $b=-11 N /\left(48 \pi^{2}\right)$ is the coefficient of $g^{3}$ in the usual QCD $\beta$ function without fermions.

\section{PT GLUON SELF-ENERGY IN THE COULOMB GAUGE}

The gauge fixing term in the Coulomb gauge $(\mathrm{CG})$ is given by

$$
\mathcal{L}=-\frac{1}{2 \xi_{C}}\left(\partial^{i} A_{i}^{a}\right)^{2}
$$

Then, with a unit vector $n^{\mu}=(1,0,0,0)$, the CG gluon propagator, $i D_{a b(\mathrm{CG})}^{\mu \nu}=i \delta_{a b} D_{(\mathrm{CG})}^{\mu \nu}$, and its inverse are expressed as

$$
\begin{aligned}
D_{(\mathrm{CG})}^{\mu \nu}(k)= & -\frac{1}{k^{2}}\left[g^{\mu \nu}+\left(1-\xi_{C} \frac{k^{2}}{\mathbf{k}^{2}}\right) \frac{k^{\mu} k^{\nu}}{\mathbf{k}^{2}}\right. \\
& \left.-\frac{k_{0}}{\mathbf{k}^{2}}\left(k^{\mu} n^{\nu}+n^{\mu} k^{\nu}\right)\right], \\
{\left[D_{(\mathrm{CG})}^{-1}\right]^{\mu \nu}(k)=} & -k^{2}\left[g^{\mu \nu}-\frac{k^{\mu} k^{\nu}}{k^{2}}\right] \\
& +\frac{1}{\xi_{C}}\left[k^{\mu} k^{\nu}-k_{0}\left(k^{\mu} n^{\nu}+n^{\mu} k^{\nu}\right)+k_{0}^{2} n^{\mu} n^{\nu}\right] .
\end{aligned}
$$


The three-gluon vertex is the same as in the FG, that is, $\Gamma_{\lambda \mu \nu}^{a b c}(p, k, q)$ in Eq. (19), and the ghost propagator $i \delta^{a b} G_{(\mathrm{CG})}$ and the ghost-gluon vertex $\Gamma_{\mu(\mathrm{CG})}^{a b c}(p, k, q)$ [see Fig. 5(a)] in the $\mathrm{CG}$ are given by

$$
\begin{gathered}
G_{(\mathrm{CG})}(k)=\frac{1}{\mathbf{k}^{2}}, \\
\Gamma_{\mu(\mathrm{CG})}^{a b c}(p, k, q)=g f^{a b c}\left[p_{\mu}-p_{0} n_{\mu}\right] .
\end{gathered}
$$

In the limit $\xi_{C}=0, D_{(\mathrm{CG})}^{\mu \nu}(k)$ reduces to the well-known form [20]

$$
D_{(\mathrm{CG})}^{00}=\frac{1}{\mathbf{k}^{2}}, \quad D_{(\mathrm{CG})}^{0 i}=0, \quad D_{(\mathrm{CG})}^{i j}=\frac{1}{k^{2}}\left(\delta^{i j}-\frac{k^{i} k^{j}}{\mathbf{k}^{2}}\right) .
$$

However, its inverse does not exist in this limit. The oneloop CG gluon self-energy was calculated in Ref. [13] in the $\xi_{C}=0$ limit using the gluon propagator in Eq. (32).

In the framework of PT, we need to use the identities in Eq. (3), satisfied by the gluon propagator and its inverse, to extract from $T_{P}$ the pinch contributions to the gluon selfenergy. Therefore, in principle, we must work with a nonzero $\xi_{C}$. Thus we recalculate the one-loop gluon self-energy in the CG with an arbitrary gauge parameter $\xi_{C}$. The results for the contributions from Fig. 1(b), the tadpole diagram [Fig. 1(c)], and the ghost diagram [Fig. 1(d)] are, respectively, as follows:

$$
\begin{aligned}
& \Pi_{(a)(\mathrm{CG})}^{\mu \nu}(k)=\frac{N}{2} g^{2} \int d p \frac{1}{p^{2} q^{2}}\left[g^{\mu \nu}\left\{8 k^{2}-\left[\left(\frac{k^{2}\left(k^{2}-2 q^{2}-4 \mathbf{k} \cdot \mathbf{p}\right)+q^{4}}{\mathbf{p}^{2}}+p^{2}\right)+(p \leftrightarrow q)\right]\right\}+\left\{p ^ { \mu } p ^ { \nu } \left[-3+\frac{(\mathbf{p} \cdot \mathbf{q})^{2}}{\mathbf{p}^{2} \mathbf{q}^{2}}+\frac{4 \mathbf{p} \cdot \mathbf{q}}{\mathbf{p}^{2}}\right.\right.\right. \\
& \left.\left.-\frac{q^{2}}{\mathbf{p}^{2} \mathbf{q}^{2}}\left(3 p^{2}+2 q^{2}+4 \mathbf{p}^{2}+\mathbf{q}^{2}+4 p_{0} q_{0}\right)\right]+(p \leftrightarrow q)\right\}+\left(p^{\mu} q^{\nu}+q^{\mu} p^{\nu}\right)\left[-5-\frac{(\mathbf{p} \cdot \mathbf{q})^{2}}{\mathbf{p}^{2} \mathbf{q}^{2}}+2(\mathbf{p} \cdot \mathbf{q})\left(\frac{1}{\mathbf{p}^{2}}+\frac{1}{\mathbf{q}^{2}}\right)\right. \\
& \left.-\frac{p^{2} q^{2}}{\mathbf{p}^{2} \mathbf{q}^{2}}\right]+\left\{( n ^ { \mu } p ^ { \nu } + p ^ { \mu } n ^ { \nu } ) \frac { 1 } { \mathbf { p } ^ { 2 } \mathbf { q } ^ { 2 } } \left[k q\left(p^{2} q_{0}-q^{2} p_{0}-2 \mathbf{p} \cdot \mathbf{q}\left(p_{0}-q_{0}\right)\right)-4 k_{0} p_{0} q_{0}(p q)\right.\right. \\
& \left.\left.\left.-\left(q^{2} \mathbf{p}^{2} q_{0}+p^{2} \mathbf{q}^{2} p_{0}-\mathbf{p} \cdot \mathbf{q}\left(p^{2} q_{0}+q^{2} p_{0}\right)\right)+p^{2} q^{2} q_{0}+q^{2} \mathbf{q}^{2} p_{0}\right]+(p \leftrightarrow q)\right\}+n^{\mu} n^{\nu} \frac{2 p_{0} q_{0}}{\mathbf{p}^{2} \mathbf{q}^{2}}\left(-2 k^{2}(p q)-p^{2} q^{2}\right)\right] \\
& +\xi_{C} \frac{N}{2} g^{2} \int d p\left[g^{\mu \nu}\left\{\left(-k^{4} \frac{1}{q^{2} \mathbf{p}^{4}}+k^{2} \frac{2}{\mathbf{p}^{4}}-\frac{q^{2}}{\mathbf{p}^{4}}\right)+(p \leftrightarrow q)\right\}+\left\{p ^ { \mu } p ^ { \nu } \left[\frac { 1 } { p ^ { 2 } \mathbf { p } ^ { 2 } \mathbf { q } ^ { 4 } } \left(k^{2} \mathbf{p}^{2}-(k q)^{2}-2(k p) p^{2}\right.\right.\right.\right. \\
& \left.\left.\left.-2 k_{0} p_{0}(k q)\right)+\frac{1}{q^{2} \mathbf{p}^{4} \mathbf{q}^{2}}\left(k^{2} \mathbf{q}^{2}-(k q)^{2}+2 k_{0} q_{0}(k q)\right)-\frac{2}{\mathbf{p}^{4}}-\frac{1}{\mathbf{q}^{4}}\right]+(p \leftrightarrow q)\right\}+\left(p^{\mu} q^{\nu}+q^{\mu} p^{\nu}\right) \\
& \times\left[\left(\frac{k^{2} \mathbf{q}^{2}+(k p)(k q)+k_{0} q_{0}\left(p^{2}-q^{2}\right)}{q^{2} \mathbf{p}^{4} \mathbf{q}^{2}}+\frac{k q}{\mathbf{p}^{4} \mathbf{q}^{2}}-\frac{2}{\mathbf{p}^{4}}\right)+(p \leftrightarrow q)\right]+\left\{( n ^ { \mu } p ^ { \nu } + p ^ { \mu } n ^ { \nu } ) \frac { k q } { \mathbf { p } ^ { 2 } \mathbf { q } ^ { 2 } } \left[k^{2}\left(\frac{q_{0}}{q^{2} \mathbf{p}^{2}}-\frac{p_{0}}{p^{2} \mathbf{q}^{2}}\right)\right.\right. \\
& \left.\left.\left.-\left(\frac{q_{0}}{\mathbf{p}^{2}}-\frac{p_{0}}{\mathbf{q}^{2}}\right)\right]+(p \leftrightarrow q)\right\}\right]+\xi_{C}^{2} \frac{N}{2} g^{2} \int d p \frac{1}{\mathbf{p}^{4} \mathbf{q}^{4}}\left[\left\{(k q)^{2} p^{\mu} p^{\nu}+(p \leftrightarrow q)\right\}-(k p)(k q)\left(p^{\mu} q^{\nu}+q^{\mu} p^{\nu}\right)\right], \\
& \Pi_{(b)(\mathrm{CG})}^{\mu \nu}(k)=\frac{N}{2} g^{2} \int d p\left[g^{\mu \nu}\left\{\frac{1}{\mathbf{p}^{2}}-\frac{1}{p^{2}}+(p \leftrightarrow q)\right\}+\left\{p^{\mu} p^{\nu} \frac{1}{p^{2} \mathbf{p}^{2}}+(p \leftrightarrow q)\right\}+\left\{\left(n^{\mu} p^{\nu}+p^{\mu} n^{\nu}\right) \frac{-p_{0}}{p^{2} \mathbf{p}^{2}}+(p \leftrightarrow q)\right\}\right] \\
& +\xi_{C} \frac{N}{2} g^{2} \int d p\left[g^{\mu \nu}\left(\frac{p^{2}}{\mathbf{p}^{4}}+(p \leftrightarrow q)\right)+p^{\mu} p^{\nu}\left(\frac{-1}{\mathbf{p}^{4}}+(p \leftrightarrow q)\right)\right], \\
& \Pi_{\text {ghost }(\mathrm{CG})}^{\mu \nu}(k)=\frac{N}{2} g^{2} \int d p \frac{1}{\mathbf{p}^{2} \mathbf{q}^{2}}\left[\left(p^{\mu} q^{\nu}+q^{\mu} p^{\nu}\right)-\left(q_{0}\left(n^{\mu} p^{\nu}+p^{\mu} n^{\nu}\right)+(p \leftrightarrow q)\right)+n^{\mu} n^{\nu} 2 p_{0} q_{0}\right],
\end{aligned}
$$

where we have chosen the variables as $k+p+q=0$ and, therefore, the integrands can be written in the forms which are symmetric in the variables $p$ and $q$. Here and in the following, the notation $+(p \leftrightarrow q)$ implies symmetrization of the preceding term under interchange of $p$ and $q$. The one-loop CG gluon self-energy is given by the sum

$$
\Pi_{(\mathrm{CG})}^{\mu \nu}=\Pi_{(a)(\mathrm{CG})}^{\mu \nu}+\Pi_{(b)(\mathrm{CG})}^{\mu \nu}+\Pi_{\text {ghost }(\mathrm{CG})}^{\mu \nu} .
$$

We have checked that the $\xi_{C}$-independent part of $\Pi_{(\mathrm{CG})}^{\mu \nu}$ agrees with the results given in Eqs. (4.6), (4.8), (4.10), and (4.12) of Ref. [13].

We now calculate the pinch contributions to the CG gluon self-energy. Since the CG gluon propagator and its inverse satisfy the relations in Eq. (3), that is, 


$$
\begin{gathered}
D_{\alpha \mu}^{(\mathrm{CG})}(k)\left[D_{(\mathrm{CG})}^{-1}\right]^{\mu \beta}(k)=D_{\alpha \mu}^{(\mathrm{CG})}(k)\left[-k^{2} d^{\mu \beta}\right]+\frac{k_{\alpha}}{\mathbf{k}^{2}}\left(k_{0} n^{\beta}-k^{\beta}\right), \\
D_{(\mathrm{CG}) \alpha \mu}^{-1}(k) D_{(\mathrm{CG})}^{\mu \beta}(k)=\left[-k^{2} d_{\alpha \mu}\right] D_{(\mathrm{CG})}^{\mu \beta}(k)+\left(k_{0} n_{\alpha}-k_{\alpha}\right) \frac{k^{\beta}}{\mathbf{k}^{2}},
\end{gathered}
$$

we can follow the prescription explained in Sec. II to extract the one-loop pinch contributions. The individual contributions in CG from the vertex (first and second kind) and box diagrams are presented in Appendix A 1. In total the pinch contribution to the CG gluon self-energy is expressed as

$$
\begin{aligned}
\Pi_{P(\mathrm{CG})}^{\mu \nu}(k)= & \frac{N}{2} g^{2} k^{2} d^{\mu \nu} \int d p \frac{1}{p^{2} q^{2}}\left[\frac{k^{2}-q^{2}-4 \mathbf{k} \cdot \mathbf{p}}{\mathbf{p}^{2}}+(p \leftrightarrow q)\right]+\frac{N}{2} g^{2} k^{2} d^{\mu \alpha} d^{\nu \beta} \int d p \frac{1}{p^{2} q^{2} \mathbf{p}^{2} \mathbf{q}^{2}}\left\{p_{\alpha} p_{\beta}\left(k^{2}+4 \mathbf{p} \cdot \mathbf{q}\right)\right. \\
& \left.+\left(p_{\alpha} n_{\beta}+n_{\alpha} p_{\beta}\right)\left[p^{2} q_{0}-q^{2} p_{0}-2 \mathbf{p} \cdot \mathbf{q}\left(p_{0}-q_{0}\right)\right]+n_{\alpha} n_{\beta} 4 p_{0} q_{0}(p q)\right\}+\frac{N}{2} g^{2}\left[d ^ { \mu \alpha } \int d p \left\{p _ { \alpha } k ^ { \nu } \left[\frac{1}{q^{2} \mathbf{p}^{2}}-\frac{1}{p^{2} \mathbf{q}^{2}}\right.\right.\right. \\
& \left.\left.\left.+\left(\frac{1}{q^{2}}-\frac{1}{p^{2}}\right) \frac{\mathbf{p} \cdot \mathbf{q}}{\mathbf{p}^{2} \mathbf{q}^{2}}\right]+n_{\alpha} k^{\nu}\left[-\frac{q_{0}}{p^{2} \mathbf{q}^{2}}-\frac{p_{0}}{q^{2} \mathbf{p}^{2}}+\left(\frac{q_{0}}{q^{2}}+\frac{p_{0}}{p^{2}}\right) \frac{\mathbf{p} \cdot \mathbf{q}}{\mathbf{p}^{2} \mathbf{q}^{2}}\right]\right\}+(\mu \leftrightarrow \nu)\right]+\xi_{C} \frac{N}{2} g^{2} \\
& \times\left[k^{2} d^{\mu \nu} \int d p\left\{k^{2}\left(\frac{1}{q^{2} \mathbf{p}^{4}}+\frac{1}{p^{2} \mathbf{q}^{4}}\right)-\frac{1}{\mathbf{p}^{4}}-\frac{1}{\mathbf{q}^{4}}\right\}+k^{2} d^{\mu \alpha} d^{\nu \beta} \int d p \frac{1}{\mathbf{p}^{2} \mathbf{q}^{2}}\left\{p_{\alpha} p_{\beta}\left[k^{2}\left(\frac{1}{p^{2} \mathbf{q}^{2}}+\frac{1}{q^{2} \mathbf{p}^{2}}\right)-\frac{2}{\mathbf{p}^{2}}-\frac{2}{\mathbf{q}^{2}}\right]\right.\right. \\
& \left.\left.+\left(p_{\alpha} n_{\beta}+n_{\alpha} p_{\beta}\right)\left[\left(\frac{p_{0}}{\mathbf{q}^{2}}-\frac{q_{0}}{\mathbf{p}^{2}}\right)-k^{2}\left(\frac{p_{0}}{p^{2} \mathbf{q}^{2}}-\frac{q_{0}}{q^{2} \mathbf{p}^{2}}\right)\right]\right\}+\left\{d^{\mu \alpha} \int d p \frac{p_{\alpha} k^{\nu}}{\mathbf{p}^{2} \mathbf{q}^{2}}\left(\frac{\mathbf{k} \cdot \mathbf{p}}{\mathbf{q}}-\frac{\mathbf{k} \cdot \mathbf{q}}{\mathbf{p}^{2}}\right)+(\mu \leftrightarrow \nu)\right\}\right] \\
& +\xi_{C}^{2} \frac{N}{2} g^{2} k^{4} d^{\mu \alpha} d^{\nu \beta} \int d p \frac{-p_{\alpha} p_{\beta}}{\mathbf{p}^{4} \mathbf{q}^{4}} .
\end{aligned}
$$

In order to compare the above result with $\Pi_{(\mathrm{CG})}^{\mu \nu}$, it is better to express Eq. (38) in terms of symmetric tensor $g^{\mu \nu}$, $p^{\mu} p^{\nu}, q^{\mu} q^{\nu},\left(p^{\mu} q^{\nu}+q^{\mu} p^{\nu}\right),\left(n^{\mu} p^{\nu}+p^{\mu} n^{\nu}\right),\left(n^{\mu} q^{\nu}+q^{\mu} n^{\nu}\right)$, and $n^{\mu} n^{\nu}$. For that purpose, we first write Eq. (38) in terms of $g^{\mu \nu}$ and symmetric tensors made up of $k, p$, and $n$ and then rewrite it in terms of $g^{\mu \nu}$ and symmetric tensors made up of $p, q$, and $n$. The terms proportional to $p^{\mu} p^{\nu}, k^{\mu} k^{\nu}$, and $\left(p^{\mu} k^{\nu}+k^{\mu} p^{\nu}\right)$ and to $\left(n^{\mu} p^{\nu}+p^{\mu} n^{\nu}\right)$ and $\left(n^{\mu} k^{\nu}+k^{\mu} n^{\nu}\right)$ will then be rewritten as

$$
\begin{aligned}
& \mathcal{R} k^{\mu} k^{\nu}+\mathcal{S}\left(p^{\mu} k^{\nu}+k^{\mu} p^{\nu}\right)+\mathcal{T} p^{\mu} p^{\nu} \\
& \quad=(\mathcal{R}-2 \mathcal{S}+\mathcal{T}) p^{\mu} p^{\nu}+(\mathcal{R}-\mathcal{S})\left(p^{\mu} q^{\nu}+q^{\mu} p^{\nu}\right)+\mathcal{R} q^{\mu} q^{\nu} \\
& \mathcal{U}\left(n^{\mu} p^{\nu}+p^{\mu} n^{\nu}\right)+\mathcal{V}\left(n^{\mu} k^{\nu}+k^{\mu} n^{\nu}\right) \\
& \quad=(\mathcal{U}-\mathcal{V})\left(n^{\mu} p^{\nu}+p^{\mu} n^{\nu}\right)-\mathcal{V}\left(n^{\mu} q^{\nu}+q^{\mu} n^{\nu}\right) .
\end{aligned}
$$

The final expression for $\Pi_{P(\mathrm{CG})}^{\mu \nu}$ is given in Appendix A 2 .

From Eq. (A4) we find that the one-loop pinch contributions are also $\xi_{C}$ dependent and these $\xi_{C}$-dependent parts exactly cancel against the $\xi_{C}$-dependent parts of $\Pi_{(\mathrm{CG})}^{\mu \nu}$. Furthermore it is easy to see that adding the $\xi_{C}$-independent parts of $\Pi_{(\mathrm{CG})}^{\mu \nu}$ and $\Pi_{P(\mathrm{CG})}^{\mu \nu}$, we obtain

$$
\begin{aligned}
\widetilde{\Pi}^{\mu \nu}(k)= & \Pi_{(\mathrm{CG})}^{\mu \nu}(k)+\Pi_{P(\mathrm{CG})}^{\mu \nu}(k) \\
= & N g^{2} \int d p \frac{1}{p^{2} q^{2}}\left[\left(4 k^{2}-p^{2}-q^{2}\right) g^{\mu \nu}\right. \\
& \left.-3\left(p^{\mu} p^{\nu}+q^{\mu} q^{\nu}\right)-5\left(p^{\mu} q^{\nu}+q^{\mu} p^{\nu}\right)\right],
\end{aligned}
$$

which is equivalent to $\hat{\Pi}^{\mu \nu}(k)$ in Eq. (25). Thus we have shown explicitly that the CG gluon self-energy $\Pi_{(\mathrm{CG})}^{\mu \nu}$ and the pinch contribution $\Pi_{P(\mathrm{CG})}^{\mu \nu}$, when combined, give the universal effective gluon self-energy $\hat{\Pi}^{\mu \nu}(k)$.

We now examine the structure of $\Pi_{P(\mathrm{CG})}^{\mu \nu}$ and discuss some of the properties of the CG gluon self-energy itself. Only the $\xi_{C}$-independent parts will be considered. First, $\Pi_{P(\mathrm{CG})}^{\mu \nu}$ is not transverse. In fact, we easily obtain, from Eq. (38),

$$
\begin{aligned}
\Pi_{P(\mathrm{CG})}^{\mu \nu} k_{\nu}= & \frac{N}{2} g^{2} k^{2} d^{\mu \alpha} \int d p\left\{p _ { \alpha } \left[\frac{1}{q^{2} \mathbf{p}^{2}}-\frac{1}{p^{2} \mathbf{q}^{2}}\right.\right. \\
& \left.+\left(\frac{1}{q^{2}}-\frac{1}{p^{2}}\right) \frac{\mathbf{p} \cdot \mathbf{q}}{\mathbf{p}^{2} \mathbf{q}^{2}}\right]+n_{\alpha}\left[-\frac{q_{0}}{p^{2} \mathbf{q}^{2}}-\frac{p_{0}}{q^{2} \mathbf{p}^{2}}\right. \\
& \left.\left.+\left(\frac{q_{0}}{q^{2}}+\frac{p_{0}}{p^{2}}\right) \frac{\mathbf{p} \cdot \mathbf{q}}{\mathbf{p}^{2} \mathbf{q}^{2}}\right]\right\},
\end{aligned}
$$

where we have used $d^{\mu \nu} k_{\nu}=0$ and $d^{\nu \beta} k_{\nu}=0$. Since the sum $\hat{\Pi}^{\mu \nu}(k)$ satisfies the transversality relation [see Eq. (26)], this means that the CG gluon self-energy is not transverse either, i.e., $\Pi_{(\mathrm{CG})}^{\mu \nu} k_{\nu} \neq 0$, which was indeed pointed out in Ref. [13].

Next, let us analyze $\Pi_{P(\mathrm{CG})}^{\mu \nu}$ in the context of hot QCD. The hard thermal loop $\delta \Pi^{\mu \nu}$ in the gluon self-energy $\Pi^{\mu \nu}$ is the piece proportional to $T^{2}$, which is the leading term in the high-temperature expansion $\left(T \gg|\mathbf{k}|\right.$ and $\left.T \gg\left|k_{0}\right|\right)$ and is generated by a small part of the integration region in one-loop 
diagrams with hard momenta of order $T$ [14]. It is known that $\delta \Pi^{\mu \nu}$ is gauge independent and satisfies the transversality relation (the Ward identity) $k^{\mu} \delta \Pi^{\mu \nu}(k)=0$ [21]. Now $\Pi^{\mu \nu}$ has a dimension of mass ${ }^{2}$ and, apart from the tensorial factors, is composed of nonsingular functions of $|\mathbf{k}|$ and $k_{0}$. Then look at the structure of $\Pi_{P(\mathrm{CG})}^{\mu \nu}$ in Eq. (38) (see also $\xi_{C}$-dependent parts). It is made up of the terms proportional to $k^{2} d^{\mu \nu}, k^{2} d^{\mu \alpha} d^{\nu \beta}$, and $d^{\mu \alpha} k^{\nu}$. The $d^{\mu \alpha} k^{\nu}$ terms appear as a result of using the formula in Eq. (11), that is, $k^{2} d^{\mu \alpha} p_{j}^{\nu}=k^{2} d^{\mu \alpha} d^{\nu \tau} p_{j \tau}+d^{\mu \alpha} k^{\nu}\left(k p_{j}\right)$. So by a simple dimensional analysis, we easily see that there is no way for the pinch contribution $\Pi_{P(\mathrm{CG})}^{\mu \nu}$ to produce a $T^{2}$ term. This means that $\Pi_{P(\mathrm{CG})}^{\mu \nu}$ does not contribute to the hard thermal loop $\delta \Pi^{\mu \nu}$. These arguments can be applied to the pinch contributions to the gluon self-energy calculated in any gauge (see Sec. V for the TAG calculation). It is clear from the discussion in Sec. II that by construction, the terms in the pinch parts always carry such factors as $k^{2} d^{\mu \nu}, k^{2} d^{\mu \alpha}, k^{4} d^{\mu \nu}$, and $k^{4} d^{\mu \alpha} d^{\nu \beta}$, and hence they do not generate a $T^{2}$ term. The gluon self-energy calculated in any gauge, when combined with the pinch contribution, gives the universal and thus gauge-independent $\hat{\Pi}^{\mu \nu}(k)$. As the pinch part does not contribute to the hard thermal loop $\delta \Pi^{\mu \nu}, \delta \Pi^{\mu \nu}$ should be gauge independent. Moreover, $\delta \Pi^{\mu \nu}$ should satisfy the transversality relation $k^{\mu} \delta \Pi^{\mu \nu}(k)=0$ since $\hat{\Pi}^{\mu \nu}(k)$ does. This is an explanation for the gauge independence and the transverse nature of the hard thermal loop $\delta \Pi^{\mu \nu}$ from the PT point of view.

In a similar way we can argue for the gauge independence of the electric mass $m_{\mathrm{el}}$ and "effective gluon mass" $m_{G}$ in hot QCD. From the expression of $\Pi_{P(C G)}^{\mu \nu}$ in Eq. (38), we see that its $(00)$ component at $k_{0}=0, \Pi_{P(\mathrm{CG})}^{00}\left(k_{0}=0,|\mathbf{k}|\right)$, vanishes in the limit $|\mathbf{k}| \rightarrow 0$. This is true for the one-loop pinch contributions calculated in any gauge, since, by construction, $\Pi_{P}^{00}$ s s (more generally $\Pi_{P}^{\mu \nu}$ ) are proportional to $k^{2}$. Thus

$$
\lim _{|\mathbf{k}| \rightarrow 0} \Pi_{P}^{00}\left(k_{0}=0,|\mathbf{k}|\right)=0 .
$$

On the other hand, the limit $|\mathbf{k}| \rightarrow 0$ of $\Pi_{(\mathrm{CG})}^{00}\left(k_{0}=0,|\mathbf{k}|\right)$ remains finite. Hence the limit

$$
\begin{aligned}
\lim _{|\mathbf{k}| \rightarrow 0} \Pi_{(\mathrm{CG})}^{00}\left(k_{0}=0,|\mathbf{k}|\right) & =\frac{1}{3} N g^{2} T^{2} \\
& =m_{\mathrm{el}}^{2}
\end{aligned}
$$

is a gauge-independent quantity. The inverse of electric mass $m_{\mathrm{el}}$ represents the screening length for static electric fields. Another example is provided by a combination of pinch contributions $(1 / 2)\left(\left(k^{2} / \mathbf{k}^{2}\right) \Pi_{P}^{00}-\Pi_{P}^{\mu \nu} g_{\mu \nu}\right)$ calculated in any gauge. Obviously the combination is proportional to $k^{2}$ and thus its limit as $k^{2} \rightarrow 0$ is 0 . Therefore, the limit

$$
\begin{aligned}
m_{G}^{2} & =\lim _{k^{2} \rightarrow 0} \frac{1}{2}\left(\frac{k^{2}}{\mathbf{k}^{2}} \Pi_{(\mathrm{CG})}^{00}-\Pi_{(\mathrm{CG})}^{\mu \nu} g_{\mu \nu}\right) \\
& =\frac{1}{6} N g^{2} T^{2}
\end{aligned}
$$

is a gauge independent quantity and is called "effective gluon mass" squared.

\section{PT GLUON SELF-ENERGY IN THE TEMPORAL AXIAL GAUGE}

The gauge fixing term in the temporal axial gauge (TAG) is provided by

$$
\mathcal{L}=-\frac{1}{2 \xi_{A}}\left(n^{\mu} A_{\mu}^{a}\right)^{2},
$$

where $n^{\mu}=(1,0,0,0)$. The gluon propagator in the TAG, $i D_{a b(\mathrm{TAG})}^{\mu \nu}=i \delta_{a b} D_{(\mathrm{TAG})}^{\mu \nu}$, and its inverse are given by

$$
\begin{aligned}
D_{(\mathrm{TAG})}^{\mu \nu}(k)= & -\frac{1}{k^{2}}\left[g^{\mu \nu}+\left(1+\xi_{A} k^{2}\right) \frac{k^{\mu} k^{\nu}}{k_{0}^{2}}\right. \\
& \left.-\frac{1}{k_{0}}\left(k^{\mu} n^{\nu}+n^{\mu} k^{\nu}\right)\right], \\
{\left[D_{(\mathrm{TAG})}^{-1}\right]^{\mu \nu}(k)=} & -k^{2}\left(g^{\mu \nu}-\frac{k^{\mu} k^{\nu}}{k^{2}}\right)-\frac{1}{\xi_{A}} n^{\mu} n^{\nu} .
\end{aligned}
$$

It is noted that the gauge parameter $\xi_{A}$ in the TAG has a dimension of mass ${ }^{-2}$. The three-gluon vertex is given again by $\Gamma_{\lambda \mu \nu}^{a b c}(p, k, q)$ in Eq. (19), and the ghost propagator $i \delta^{a b} G_{(\mathrm{TAG})}$ and the ghost-gluon vertex $\Gamma_{\mu(\mathrm{TAG})}^{a b c}(p, k, q)[$ see Fig. 5(b)] in TAG are, respectively,

$$
G_{(\mathrm{TAG})}(k)=\frac{-i}{k_{0}},
$$

$$
\Gamma_{\mu(\mathrm{TAG})}^{a b c}(p, k, q)=g f^{a b c}\left[-i n_{\mu}\right]
$$

The one-loop gluon self-energy in the TAG was calculated in Refs. $[12,13]$ in the $\xi_{A}=0$ limit. There, the ghost loop contribution was omitted due to the argument that the ghost field decouples in this limit. However, in the limit $\xi_{A}=0$ the inverse of the gluon propagator does not exist. So in the framework of PT we work with a nonzero $\xi_{A}$. We recalculate the gluon self-energy using the gluon propagator with an arbitrary $\xi_{A}$ given in Eq. (46). For a nonzero $\xi_{A}$ the ghost should be taken into account and at one-loop level it contributes to the $\xi_{A}$-independent part of $\Pi_{(\mathrm{TAG})}^{00}[13]$. The contributions of Fig. 1(b), of the tadpole diagram [Fig. 1(c)], and the ghost diagram [Fig. 1(d)] are, respectively, 


$$
\begin{aligned}
& \Pi_{(a)(\mathrm{TAG})}^{\mu \nu}(k)=\frac{N}{2} g^{2} \int d p \frac{1}{p^{2} q^{2}}\left[g^{\mu \nu}\left\{8 k^{2}-\left[\left(\frac{k^{2}\left(k^{2}+2 p^{2}-q^{2}-4 \mathbf{k} \cdot \mathbf{p}\right)-p^{2} q^{2}}{p_{0}^{2}}+p^{2}\right)+(p \leftrightarrow q)\right]\right\}\right. \\
& +\left\{p^{\mu} p^{\nu}\left[-3+\frac{(\mathbf{p} \cdot \mathbf{q})^{2}}{p_{0}^{2} q_{0}^{2}}-\frac{2 \mathbf{p} \cdot \mathbf{q}}{p_{0}^{2} q_{0}^{2}}\left(q^{2}-2 q_{0}^{2}\right)+\frac{q^{4}}{p_{0}^{2} q_{0}^{2}}-\frac{p^{2}+q^{2}}{p_{0}^{2}}+\frac{q^{2}}{q_{0}^{2}}\right]+(p \leftrightarrow q)\right\} \\
& +\left(p^{\mu} q^{\nu}+q^{\mu} p^{\nu}\right)\left[-5-\frac{(\mathbf{p} \cdot \mathbf{q})^{2}}{p_{0}^{2} q_{0}^{2}}+(\mathbf{p} \cdot \mathbf{q})\left(\frac{p^{2}+q^{2}}{p_{0}^{2} q_{0}^{2}}+\frac{2}{p_{0}^{2}}+\frac{2}{q_{0}^{2}}\right)-\frac{p^{2} q^{2}}{p_{0}^{2} q_{0}^{2}}\right] \\
& +\left\{\left(n^{\mu} p^{\nu}+p^{\mu} n^{\nu}\right) \frac{1}{p_{0}^{2} q_{0}^{2}}\left[k q\left(-p^{2} q_{0}+q^{2} p_{0}-2 \mathbf{p} \cdot \mathbf{q}\left(p_{0}-q_{0}\right)\right)-4 k_{0} p_{0} q_{0}(p q)+q^{2} p_{0} q_{0}^{2}\right]+(p \leftrightarrow q)\right\} \\
& \left.+n^{\mu} n^{\nu} \frac{2 p_{0} q_{0}}{p_{0}^{2} q_{0}^{2}}\left(-2 k^{2}(p q)-p^{2} q^{2}\right)\right]+\xi_{A} \frac{N}{2} g^{2} \int d p\left[g^{\mu \nu}\left\{\left(k^{4} \frac{1}{q^{2} p_{0}^{2}}-k^{2} \frac{2}{p_{0}^{2}}+\frac{q^{2}}{p_{0}^{2}}\right)+(p \leftrightarrow q)\right\}\right. \\
& +\left\{p^{\mu} p^{\nu}\left[-k^{2}\left(\frac{1}{p^{2} q_{0}^{2}}+\frac{1}{q^{2} p_{0}^{2}}\right)+\frac{(k q)^{2}}{p_{0}^{2} q_{0}^{2}}\left(\frac{1}{p^{2}}+\frac{1}{q^{2}}\right)-\frac{2 k_{0}(k q)}{p_{0}^{2} q_{0}^{2}}\left(\frac{q_{0}}{q^{2}}-\frac{p_{0}}{p^{2}}\right)+\frac{2}{p_{0}^{2}}+\frac{1}{q_{0}^{2}}\right]+(p \leftrightarrow q)\right\} \\
& +\left(p^{\mu} q^{\nu}+q^{\mu} p^{\nu}\right)\left[\left(\frac{-k^{2} p_{0}^{2}-(k p)(k q)+k_{0} p_{0}\left(p^{2}-q^{2}\right)}{p^{2} p_{0}^{2} q_{0}^{2}}+\frac{2}{p_{0}^{2}}\right)+(p \leftrightarrow q)\right] \\
& \left.+\left\{\left(n^{\mu} p^{\nu}+p^{\mu} n^{\nu}\right) \frac{k q}{p_{0}^{2} q_{0}^{2}}\left[k^{2}\left(\frac{p_{0}}{p^{2}}-\frac{q_{0}}{q^{2}}\right)+q_{0}-p_{0}\right]+(p \leftrightarrow q)\right\}\right] \\
& +\xi_{A}^{2} \frac{N}{2} g^{2} \int d p \frac{1}{p_{0}^{2} q_{0}^{2}}\left[\left\{(k q)^{2} p^{\mu} p^{\nu}+(p \leftrightarrow q)\right\}-(k p)(k q)\left(p^{\mu} q^{\nu}+q^{\mu} p^{\nu}\right)\right], \\
& \Pi_{(b)(\mathrm{TAG})}^{\mu \nu}(k)=\frac{N}{2} g^{2} \int d p\left[g^{\mu \nu}\left\{\frac{-1}{p^{2}}+\frac{-1}{p_{0}^{2}}+(p \leftrightarrow q)\right\}+\left\{p^{\mu} p^{\nu} \frac{1}{p^{2} p_{0}^{2}}+(p \leftrightarrow q)\right\}+\left\{\left(n^{\mu} p^{\nu}+p^{\mu} n^{\nu}\right) \frac{-1}{p^{2} p_{0}}+(p \leftrightarrow q)\right\}\right] \\
& +\xi_{A} \frac{N}{2} g^{2} \int d p\left[g^{\mu \nu}\left(\frac{-p^{2}}{p_{0}^{2}}+(p \leftrightarrow q)\right)+p^{\mu} p^{\nu}\left(\frac{1}{p_{0}^{2}}+(p \leftrightarrow q)\right)\right], \\
& \Pi_{\text {ghost(TAG) }}^{\mu \nu}(k)=\frac{N}{2} g^{2} \int d p n^{\mu} n^{\nu} \frac{2}{p_{0} q_{0}} .
\end{aligned}
$$

The one-loop gluon self-energy in the TAG is then given by the sum

$$
\Pi_{(\mathrm{TAG})}^{\mu \nu}=\Pi_{(a)(\mathrm{TAG})}^{\mu \nu}+\Pi_{(b)(\mathrm{TAG})}^{\mu \nu}+\Pi_{\mathrm{ghost}(\mathrm{TAG})}^{\mu \nu}
$$

The $\xi_{A}$-independent part of $\Pi_{(\mathrm{TAG})}^{\mu \nu}$ agrees with the results given in Eqs. (4.5), (4.7), (4.9), (4.11) of Ref. [13] except for the ghost contribution to $\Pi_{(\mathrm{TAG})}^{00}$.

We now calculate the pinch contributions in the TAG. Since the TAG propagator and its inverse satisfy the relations in Eq. (3), i.e.,

$$
\begin{aligned}
& D_{\alpha \mu}^{(\mathrm{TAG})}(k)\left[D_{(\mathrm{TAG})}^{-1}\right]^{\mu \beta}(k)=D_{\alpha \mu}^{(\mathrm{TAG})}(k)\left[-k^{2} d^{\mu \beta}\right]+k_{\alpha}\left(\frac{n^{\beta}}{k_{0}}-\frac{k^{\beta}}{k^{2}}\right), \\
& D_{(\mathrm{TAG}) \alpha \mu}^{-1}(k) D_{(\mathrm{TAG})}^{\mu \beta}(k)=\left[-k^{2} d_{\alpha \mu}\right] D_{(\mathrm{TAG})}^{\mu \beta}(k)+\left(\frac{n_{\alpha}}{k_{0}}-\frac{k_{\alpha}}{k^{2}}\right) k^{\beta},
\end{aligned}
$$

we can follow the same procedure as before and we obtain, for the pinch contribution to the gluon self-energy in the TAG, 


$$
\begin{aligned}
\Pi_{P(\mathrm{TAG})}^{\mu \nu}(k)= & \frac{N}{2} g^{2} k^{2} d^{\mu \nu} \int d p \frac{1}{p^{2} q^{2}}\left[\frac{k^{2}+2 p^{2}-q^{2}-4 \mathbf{k} \cdot \mathbf{p}}{p_{0}^{2}}+(p \leftrightarrow q)\right]+\frac{N}{2} g^{2} k^{2} d^{\mu \alpha} d^{\nu \beta} \int d p \frac{1}{p^{2} q^{2} p_{0}^{2} q_{0}^{2}} \\
& \times\left\{p_{\alpha} p_{\beta}\left(4 p_{0} q_{0}-k^{2}\right)+\left(p_{\alpha} n_{\beta}+n_{\alpha} p_{\beta}\right)\left[-p^{2} q_{0}+q^{2} p_{0}-2 \mathbf{p} \cdot \mathbf{q}\left(p_{0}-q_{0}\right)\right]+n_{\alpha} n_{\beta} 4 p_{0} q_{0}(p q)\right\} \\
& +\xi_{A} \frac{N}{2} g^{2}\left[k^{2} d^{\mu \nu} \int d p\left\{-k^{2}\left(\frac{1}{q^{2} p_{0}^{2}}+\frac{1}{p^{2} q_{0}^{2}}\right)+\frac{1}{p_{0}^{2}}+\frac{1}{q_{0}^{2}}\right\}+k^{2} d^{\mu \alpha} d^{\nu \beta} \int d p \frac{1}{p_{0}^{2} q_{0}^{2}}\left\{p_{\alpha} p_{\beta}\left[-k^{2}\left(\frac{1}{p^{2}}+\frac{1}{q^{2}}\right)\right]\right.\right. \\
& \left.\left.+\left(p_{\alpha} n_{\beta}+n_{\alpha} p_{\beta}\right)\left[q_{0}-p_{0}+k^{2}\left(\frac{p_{0}}{p^{2}}-\frac{q_{0}}{q^{2}}\right)\right]\right\}\right]+\xi_{A}^{2} \frac{N}{2} g^{2} k^{4} d^{\mu \alpha} d^{\nu \beta} \int d p \frac{-p_{\alpha} p_{\beta}}{p_{0}^{2} q_{0}^{2}} .
\end{aligned}
$$

The individual contributions in the TAG from the vertex (first and second kind) and box diagrams are presented in Appendix B 1.

The expression of $\Pi_{P(\mathrm{TAG})}^{\mu \nu}$ is further rewritten in terms of symmetric tensors $g^{\mu \nu}, p^{\mu} p^{\nu}, q^{\mu} q^{\nu}, \quad\left(p^{\mu} q^{\nu}+q^{\mu} p^{\nu}\right)$, $\left(n^{\mu} p^{\nu}+p^{\mu} n^{\nu}\right),\left(n^{\mu} q^{\nu}+q^{\mu} n^{\nu}\right)$, and $n^{\mu} n^{\nu}$. The result is given in Appendix B 2. From this expression we can see that the one-loop pinch contributions are also $\xi_{A}$ dependent and these $\xi_{A}$-dependent parts exactly cancel against the $\xi_{A}$-dependent parts of $\Pi_{(\mathrm{TAG})}^{\mu \nu}$. Also we find the sum of $\Pi_{\text {(TAG) }}^{\mu \nu}$ and $\Pi_{P(\mathrm{TAG})}^{\mu \nu}$ is equal to $\widetilde{\Pi}^{\mu \nu}$ in Eq. (40) and thus equal to the universal $\hat{\Pi}^{\mu \nu}$ in Eq. (25).

Let us now examine the results of these TAG calculations. We will only consider the $\xi_{A}$-independent part. First it is easily seen from Eq. (53) that the pinch contribution $\Pi_{P(\mathrm{TAG})}^{\mu \nu}$ is transverse, i.e., $k_{\mu} \Pi_{P(\mathrm{TAG})}^{\mu \nu}=0$. Hence the TAG gluon self-energy $\Pi_{\text {(TAG) }}^{\mu \nu}$ should be transverse [13]. Here it is noted that we have included the ghost-loop contribution in $\Pi_{(\mathrm{TAG})}^{\mu \nu}$.

At zero temperature $(T=0)$ the $\xi_{A}$-independent part of the pinch contribution $\Pi_{P(\mathrm{TAG})}^{\mu \nu}$ does not contain ultraviolet divergences. This can be easily seen from the examination of the $g^{\mu \nu}$ part of $\Pi_{P(\mathrm{TAG})}^{\mu \nu}$ in the limit $\mathbf{k}=\mathbf{0}$ (and remains true for $\mathbf{k} \neq \mathbf{0})$. Applying the projection operator $\frac{1}{3} d_{\mu \nu}$ to the $\xi_{A}$-independent part of $\Pi_{P(\mathrm{TAG})}^{\mu \nu}$, we find, in the limit $\mathbf{k}=\mathbf{0}$,

$$
\begin{aligned}
& \text { d.p. } \text { of }\left[\frac{1}{3} d_{\mu \nu} \Pi_{p(\mathrm{TAG})}^{\mu \nu}\right]_{\xi_{A}=0} \\
& \quad=\text { d.p. of }\left[\frac{N}{6} g^{2} k_{0}^{2} \int d p\left(\frac{4}{p^{2} q^{2}}+\frac{2}{q^{2} p_{0}^{2}}\right)\right]
\end{aligned}
$$

where an abbreviation "d.p. of" stands for "divergent part of" and we have dropped the $k_{0}^{2}$ terms in the numerator of the integrand which would only contribute to the finite part. Also we have replaced $q_{0}$ with $-p_{0}$ and $q$ in the numerator with $-p$, since $q=-p-k$ and these replacements do not modify the ultraviolet divergent part. As a final step we use the following two integral formulas [22]:

$$
\text { d.p. of }\left[\int d p \frac{1}{p^{2}(p+k)^{2}}\right]=\Delta \text {, }
$$

$$
\text { d.p. of }\left[\int d p \frac{1}{(p+k)^{2} p_{0}^{2}}\right]=-2 \Delta \text {, }
$$

where the loop integral $\int d p$ is defined in Eq. (16) and $\Delta=\left(1 / 16 \pi^{2}\right)[2 /(4-D)]$. Thus we find

$$
\text { d.p. of }\left[\frac{1}{3} d_{\mu \nu} \Pi_{P(\mathrm{TAG})}^{\mu \nu}\right]_{\xi_{A}=0}=0,
$$

and hence the $\xi_{A}$-independent part of $\Pi_{P(\text { TAG })}^{\mu \nu}$ is ultraviolet finite. We have shown in Sec. III that, at zero temperature, the divergent part of the universal gluon self-energy $\hat{\Pi}^{\mu \nu}$, which is the sum of $\Pi_{(\mathrm{TAG})}^{\mu \nu}$ and $\Pi_{P(\mathrm{TAG})}^{\mu \nu}$, gives us complete information on the correct running of the QCD coupling constant at one-loop level. The fact that $\Pi_{P(\mathrm{TAG})}^{\mu \nu}$ is ultraviolet finite, therefore, implies that in one-loop TAG calculations the only knowledge of the gluon self-energy is enough to determine the QCD $\beta$ function, which is indeed true for $\xi_{A}=0$ [22].

There is one subtlety in the quantization of gauge theories in the TAG $[11,16]$. Spurious singularities appear in the loop calculations. The gauge condition $n^{\mu} A_{\mu}^{a}=0$ in the TAG is not enough to fix the gauge uniquely and there still remains a freedom of time-independent gauge transformations. This residual invariance manifests itself as unphysical poles in the longitudinal part of the gluon propagator given in Eq. (46). In the TAG calculation of the gluon self-energy, these unphysical poles in the gluon propagator give spurious singularities. To circumvent these singularities, several methods have been proposed, and most noticeable are the principalvalue prescription [17], the $n_{\mu}^{*}$ prescription [18], and the $\alpha$ prescription [19].

We now know that the longitudinal part of the TAG propagator gives rise to pinch parts. Thus the spurious singularities due to the unphysical poles of the propagator also appear in the pinch contribution. Once this pinch contribution is added to the TAG gluon self-energy, the singularities due to the ill-fated unphysical poles cancel out. To illustrate how these cancellations actually occur, we present the PT calculation in the TAG of the gauge-independent thermal $\beta$ function in a hot Yang-Mills gas [6].

As stated in Sec. III, the PT modified gluon self-energy $\hat{\Pi}_{\mu \nu}$ contains the running of the QCD coupling. When the renormalization condition of the three-gluon vertex is chosen at the static and symmetric point, the thermal $\beta$ function $\beta_{T}$ is obtained through a formula [23-25] 


$$
\beta_{T} \equiv T \frac{d g(T, \kappa)}{d T}=\frac{g}{2 \kappa^{2}} T \frac{d \Pi_{\perp}(T, \kappa)}{d T},
$$

where $\Pi_{\perp}(T, \kappa)=\Pi_{\perp}\left(T, k_{0}=0, \kappa=|\mathbf{k}|\right)$ is the transverse function of the gluon self-energy $\Pi_{\mu \nu}$ at the static limit. Here for $\Pi_{\mu \nu}$ we should use $\hat{\Pi}_{\mu \nu}$, namely, the sum of the usual oneloop gluon self-energy and the pinch contribution.

In the static limit $k_{0}=0$, we have $\Pi_{\perp}(T, \kappa)$ $=\frac{1}{2} \Pi_{i i}\left(k_{0}=0, \kappa\right)$. The TAG calculation of $\Pi_{i i}\left(k_{0}=0, \kappa\right)$ was performed in Ref. [13]. After the $p_{0}$ summation and the angular integration, but before the $p(=|\mathbf{p}|)$ integration, $\Pi_{i i}^{(\mathrm{TAG})}(0, \kappa)$ is given in Eq. (4.43) of Ref. [13] as

$$
\begin{aligned}
\Pi_{i i}^{(\mathrm{TAG})}(0, \kappa)= & \frac{N g^{2}}{2 \pi^{2}} \int_{0}^{\infty} d p p n(p)\left[-2+\frac{\kappa^{2}}{p_{ \pm}^{2}}+\frac{\kappa^{4}}{4 p^{2} p_{ \pm}^{2}}\right. \\
& \left.+\left(\frac{2 p}{\kappa}+\frac{5 \kappa}{2 p}-\frac{\kappa^{3}}{2 p p_{ \pm}^{2}}-\frac{\kappa^{5}}{16 p^{3} p_{ \pm}^{2}}\right) \ln \left|\frac{2 p+\kappa}{2 p-\kappa}\right|\right] .
\end{aligned}
$$

where $n(p)=1 /[\exp (p / T)-1]$ is the Bose-Einstein statistical distribution function, and the principal value prescription was supposed to be applied for $1 / p_{ \pm}^{2}$. If we do not use the principal value prescription and replace $p_{ \pm}^{2}$ with $p^{2}$, we see that the integrand (the terms in []) would behave as $-4 \kappa^{2} / 3 p^{2}$ for small $p$.

Now let us calculate the pinch contribution to $\Pi_{\perp}(T, \kappa)$ in the TAG. Applying the projection operator

$$
t_{i j}=\frac{1}{2}\left(\delta_{i j}-\frac{k_{i} k_{j}}{\mathbf{k}^{2}}\right)
$$

to the spatial part of $\Pi_{P(\mathrm{TAG})}^{\mu \nu}$ in Eq. (53) (we are only interested in the $\xi_{A}$-independent part), we obtain, in the static limit,

$$
\begin{aligned}
\Pi_{\perp}^{P(\mathrm{TAG})}(T, \kappa)= & t_{i j} \Pi_{P(\mathrm{TAG})}^{i j}\left(k_{0}=0, \kappa\right) \\
= & -N g^{2} \kappa^{2} \int d p\left\{\frac{\mathbf{k}^{2}+4 \mathbf{k} \cdot \mathbf{p}}{p^{2} q^{2} p_{0}^{2}}+\frac{1}{p^{2} p_{0}^{2}}-\frac{2}{q^{2} p_{0}^{2}}\right\} \\
& -\frac{N}{4} g^{2} \kappa^{2} \int d p\left[\mathbf{p}^{2}-\frac{(\mathbf{k} \cdot \mathbf{p})^{2}}{\mathbf{k}^{2}}\right] \\
& \times\left\{\frac{\mathbf{k}^{2}}{p^{2} q^{2} p_{0}^{2} q_{0}^{2}}-\frac{4}{p^{2} q^{2} p_{0}^{2}}\right\},
\end{aligned}
$$

where the terms proportional to $\left(p_{\alpha} n_{\beta}+n_{\alpha} p_{\beta}\right)$ and $n_{\alpha} n_{\beta}$ in $\Pi_{P(\mathrm{TAG})}^{\mu \nu}$ do not contribute to $\Pi_{\perp}^{P(\mathrm{TAG})}$. After the $p_{0}$ summation and the angular integration, $\Pi_{\perp}^{P(\mathrm{TAG})}(T, \kappa)$ is rewritten as

$$
\begin{aligned}
\Pi_{\perp}^{P(\mathrm{TAG})}(T, \kappa)= & \frac{N g^{2}}{4 \pi^{2}} \int_{0}^{\infty} d p p n(p)\left[-\frac{\kappa^{2}}{p^{2}}-\frac{\kappa^{4}}{4 p^{4}}\right. \\
& \left.+\left(\frac{\kappa}{p}+\frac{\kappa^{3}}{2 p^{3}}+\frac{\kappa^{5}}{16 p^{5}}\right) \ln \left|\frac{2 p+\kappa}{2 p-\kappa}\right|\right] .
\end{aligned}
$$

where we have used formulas given in Appendix C. Note that the integrand behaves as $4 \kappa^{2} / 3 p^{2}$ for small $p$. When $\Pi_{\perp}^{(\mathrm{TAG})}$ and $\Pi_{\perp}^{P(\mathrm{TAG})}$ are combined [remember $\Pi_{\perp}^{(\mathrm{TAG})}=\frac{1}{2}$ $\left.\Pi_{i i}^{(\mathrm{TAG})}(0, \kappa)\right]$, the $\kappa^{2} / p^{2}$ singularities cancel and the integrand becomes regular as $p \rightarrow 0$. We can, therefore, evaluate the sum

$$
\begin{aligned}
\Pi_{\perp}(T, \kappa)= & \Pi_{\perp}^{(\mathrm{TAG})}(T, \kappa)+\Pi_{\perp}^{P(\mathrm{TAG})}(T, \kappa) \\
= & \frac{N g^{2}}{4 \pi^{2}} \int_{0}^{\infty} d p p n(p)\left[-2+\left(\frac{2 p}{\kappa}+\frac{7 \kappa}{2 p}\right)\right. \\
& \left.\times \ln \left|\frac{2 p+\kappa}{2 p-\kappa}\right|\right] .
\end{aligned}
$$

without recourse to the principal value prescription or to the other prescriptions mentioned before and obtain in the limit $\kappa \ll T$

$$
\Pi_{\perp}(T, \kappa) \approx N g^{2} \kappa T \frac{7}{16}+O\left(\kappa^{2}\right)
$$

Inserting the above expression into Eq. (58), we find for the gauge-independent thermal $\beta$ function

$$
\beta_{T}=g^{3} N \frac{7}{32} \frac{T}{\kappa},
$$

which coincides with the result of Refs. [24, 6]. What we have learned from these calculations is that spurious singularities in the TAG appear only in the gauge-dependent parts and that when we deal with physical and/or gaugeindependent quantities, these singularities cancel among themselves and disappear.

\section{SUMMARY AND DISCUSSION}

In this paper we have used the $S$-matrix PT and calculated the one-loop effective gluon self-energy in two noncovariant gauges, namely, the CG and the TAG. The oneloop gluon self-energies calculated in the CG and the TAG are different in form from each other and have complicated expressions. However, we showed explicitly that once the pinch contributions are added, they turn out to be identical and coincide with the result previously obtained with covariant gauges. Some properties of the CG and TAG gluon self-energies were discussed by simply analyzing the structure of their pinch contributions. In the context of hot QCD, we could explain the gauge-independence of the hard thermal loop $\delta \Pi^{\mu \nu}$, the electric mass $m_{\mathrm{el}}$, and the "effective gluon mass" $m_{G}$ from the PT point of view.

There appear spurious singularities in the TAG gluon selfenergy. These singularities are also present in the TAG pinch contribution. When the pinch contribution is added to the 
TAG gluon self-energy, the singularities cancel out. For an illustration of this cancellation, we calculated, in the TAG, the thermal $\beta$ function in the framework of PT. The $\beta$ function thus obtained is indeed gauge independent [6,7]. However, the result is incomplete in the following sense: as Elmfors and Kobes pointed out [25], the leading contribution to $\beta_{T}$, which gives a term $T / \kappa$, does not come from the hard part of the loop integral, responsible for a $T^{2} / \kappa^{2}$ term, but from soft loop integral. Hence it is not consistent to stop the calculation at one-loop order for soft internal momenta, and the resummed propagators and vertices [14] must be used to obtain the complete leading contribution. The PT algorithm still works even when we use the resummed propagators and vertices [26]. It can be shown that the resummed effective gluon self-energy obtained in the framework of PT is gaugeindependent and that, using this effective gluon self-energy, we can obtain the correct thermal $\beta$ function in the leading order. Also it can be shown that the resummed pinch contri- butions vanish on shell, and thus do not modify the result of Braaten and Pisarski [14] for the gluon damping rate in the leading order.

Note added in proof. After submitting this paper for publication, our attention has been called to a recent paper [27] by Papavassiliou and Pilaftsis, where the independence of the PT results on the gauge-fixing procedure and the uniqueness of the PT algorithm are discussed. We would like to thank Dr. Papavassiliou for bringing [27] to our attention.

\section{ACKNOWLEDGMENTS}

The authors would like to thank Professor A. Sirlin, Professor D. Zwanziger, Dr. M. Schaden, and Dr. K. Philippides for useful discussions. K.S. would like to thank Professor A. Sirlin for the hospitality extended to him at New York University where this work was done. This work was supported in part by Yokohama National University Foundation.

\section{APPENDIX A: COULOMB GAUGE}

\section{Pinch contribution}

(i) The contribution of the vertices of the first kind:

$$
\Pi_{P(\mathrm{CG})}^{\mu \nu\left(V_{1}\right)}=\frac{N}{2} g^{2} k^{2} d^{\mu \nu} \int d p\left(\frac{-1}{p^{2} \mathbf{p}^{2}}+\frac{-1}{q^{2} \mathbf{q}^{2}}\right)+\xi_{C} \frac{N}{2} g^{2} k^{2} d^{\mu \nu} \int d p\left(\frac{1}{\mathbf{p}^{4}}+\frac{1}{\mathbf{q}^{4}}\right) .
$$

(ii) The contribution of the vertices of the second kind:

$$
\begin{aligned}
\Pi_{P(\mathrm{CG})}^{\mu \nu\left(V_{2}\right)}= & N g^{2} k^{2} d^{\mu \nu} \int d p \frac{2}{p^{2} q^{2}}\left(\frac{-\mathbf{k} \cdot \mathbf{p}}{\mathbf{p}^{2}}+\frac{-\mathbf{k} \cdot \mathbf{q}}{\mathbf{q}^{2}}\right)+N g^{2} k^{2} d^{\mu \alpha} d^{\nu \beta} \int d p \frac{1}{p^{2} q^{2} \mathbf{p}^{2} \mathbf{q}^{2}}\left\{p_{\alpha} p_{\beta} 2 \mathbf{p} \cdot \mathbf{q}+n_{\alpha} n_{\beta} p_{0} q_{0}\left(k^{2}+2 p q\right)\right. \\
& \left.+\left(p_{\alpha} n_{\beta}+n_{\alpha} p_{\beta}\right) \frac{1}{2}\left[p_{0} p^{2}-q_{0} q^{2}+2\left(p_{0}-q_{0}\right)\left(p_{0} q_{0}-2 \mathbf{p} \cdot \mathbf{q}\right)\right]\right\}+\frac{N}{2} g^{2}\left[d ^ { \mu \alpha } \int d p \left\{p _ { \alpha } k ^ { \nu } \left[\frac{1}{q^{2} \mathbf{p}^{2}}-\frac{1}{p^{2} \mathbf{q}^{2}}\right.\right.\right. \\
& \left.\left.\left.+\left(\frac{1}{q^{2}}-\frac{1}{p^{2}}\right) \frac{\mathbf{p} \cdot \mathbf{q}}{\mathbf{p}^{2} \mathbf{q}^{2}}\right]+n_{\alpha} k^{\nu}\left[-\frac{q_{0}}{p^{2} \mathbf{q}^{2}}-\frac{p_{0}}{q^{2} \mathbf{p}^{2}}+\left(\frac{q_{0}}{q^{2}}+\frac{p_{0}}{p^{2}}\right) \frac{\mathbf{p} \cdot \mathbf{q}}{\mathbf{p}^{2} \mathbf{q}^{2}}\right]\right\}+(\mu \leftrightarrow \nu)\right]+\xi_{C} N g^{2}\left[k^{2} d^{\mu \nu} \int d p\right. \\
& \times\left\{k^{2}\left(\frac{1}{q^{2} \mathbf{p}^{4}}+\frac{1}{p^{2} \mathbf{q}^{4}}\right)-\frac{1}{\mathbf{p}^{4}}-\frac{1}{\mathbf{q}^{4}}\right\}+k^{2} d^{\mu \alpha} d^{\nu \beta} \int d p \frac{1}{\mathbf{p}^{2} \mathbf{q}^{2}}\left\{p_{\alpha} p_{\beta}\left[k^{2}\left(\frac{1}{p^{2} \mathbf{q}^{2}}+\frac{1}{q^{2} \mathbf{p}^{2}}\right)-\frac{1}{\mathbf{p}^{2}}-\frac{1}{\mathbf{q}^{2}}\right]+\left(p_{\alpha} n_{\beta}+n_{\alpha} p_{\beta}\right)\right. \\
& \left.\left.\times\left[\frac{1}{2}\left(\frac{p_{0}}{\mathbf{q}^{2}}-\frac{q_{0}}{\mathbf{p}^{2}}\right)-k^{2}\left(\frac{p_{0}}{p^{2} \mathbf{q}^{2}}-\frac{q_{0}}{q^{2} \mathbf{p}^{2}}\right)\right]\right\}+\left\{d^{\mu \alpha} \int d p \frac{p_{\alpha} k^{\nu}}{2 \mathbf{p}^{2} \mathbf{q}^{2}}\left(\frac{\mathbf{k} \cdot \mathbf{p}}{\mathbf{q}^{2}}-\frac{\mathbf{k} \cdot \mathbf{q}}{\mathbf{p}^{2}}\right)+(\mu \leftrightarrow \nu)\right\}\right] \\
& +\xi_{C}^{2} N g^{2} k^{4} d^{\mu \alpha} d^{\nu \beta} \int d p \frac{-p_{\alpha} p_{\beta}}{\mathbf{p}^{4} \mathbf{q}^{4}} .
\end{aligned}
$$

(iii) The box contribution:

$$
\begin{aligned}
\Pi_{P(\mathrm{CG})}^{\mu \nu(\mathrm{box})=} & \frac{N}{2} g^{2} k^{4} d^{\mu \nu} \int d p \frac{1}{p^{2} q^{2}}\left(\frac{1}{\mathbf{p}^{2}}+\frac{1}{\mathbf{q}^{2}}\right)+\frac{N}{2} g^{2} k^{4} d^{\mu \alpha} d^{\nu \beta} \int d p \frac{p_{\alpha} p_{\beta}+\left(p_{\alpha} n_{\beta}+n_{\alpha} p_{\beta}\right)\left(q_{0}-p_{0}\right)-2 n_{\alpha} n_{\beta} p_{0} q_{0}}{p^{2} q^{2} \mathbf{p}^{2} \mathbf{q}^{2}} \\
& +\xi_{C} \frac{N}{2} g^{2} k^{4}\left[d^{\mu \nu} \int d p\left(\frac{-1}{q^{2} \mathbf{p}^{4}}+\frac{-1}{p^{2} \mathbf{q}^{4}}\right)+d^{\mu \alpha} d^{\nu \beta} \int d p\left\{-\frac{p_{\alpha} p_{\beta}}{\mathbf{p}^{2} \mathbf{q}^{2}}\left(\frac{1}{p^{2} \mathbf{q}^{2}}+\frac{1}{q^{2} \mathbf{p}^{2}}\right)+\frac{p_{\alpha} n_{\beta}+n_{\alpha} p_{\beta}}{\mathbf{p}^{2} \mathbf{q}^{2}}\left(\frac{p_{0}}{p^{2} \mathbf{q}^{2}}-\frac{q_{0}}{q^{2} \mathbf{p}^{2}}\right)\right\}\right] \\
& +\xi_{C}^{2} \frac{N}{2} g^{2} k^{4} d^{\mu \alpha} d^{\nu \beta} \int d p \frac{p_{\alpha} p_{\beta}}{\mathbf{p}^{4} \mathbf{q}^{4}} .
\end{aligned}
$$




\section{Expression of $\Pi_{P(\mathbf{C G})}^{\mu \nu}$}

The pinch contribution to the gluon self-energy in the CG is rewritten in terms of symmetric tensors $g_{\mu \nu}, p_{\mu} p_{\nu}, q_{\mu} q_{\nu}$, $\left(p_{\mu} q_{\nu}+q_{\mu} p_{\nu}\right),\left(n_{\mu} p_{\nu}+p_{\mu} n_{\nu}\right),\left(n_{\mu} q_{\nu}+q_{\mu} n_{\nu}\right)$, and $n_{\mu} n_{\nu}:$

$$
\begin{aligned}
& \Pi_{P(\mathrm{CG})}^{\mu \nu}(k)=\frac{N}{2} g^{2} \int d p \frac{1}{p^{2} q^{2} \mathbf{p}^{2} \mathbf{q}^{2}}\left[g^{\mu \nu} k^{2}\left\{\mathbf{q}^{2}\left(k^{2}-q^{2}-4 \mathbf{k} \cdot \mathbf{p}\right)+(p \leftrightarrow q)\right\}+\left\{p ^ { \mu } p ^ { \nu } \left[-(\mathbf{p} \cdot \mathbf{q})^{2}-4(\mathbf{p} \cdot \mathbf{q}) \mathbf{q}^{2}+4 p_{0} q_{0} q^{2}+q^{4}\right.\right.\right. \\
& \left.\left.+4 \mathbf{p}^{2} q^{2}+3 p^{2} q^{2}-3 \mathbf{p}^{2} \mathbf{q}^{2}\right]+(p \leftrightarrow q)\right\}+\left(p^{\mu} q^{\nu}+q^{\mu} p^{\nu}\right)\left\{(\mathbf{p} \cdot \mathbf{q})^{2}-2(\mathbf{p} \cdot \mathbf{q})\left(\mathbf{p}^{2}+\mathbf{q}^{2}\right)-5 \mathbf{p}^{2} \mathbf{q}^{2}\right\}+\left\{\left(n^{\mu} p^{\nu}+p^{\mu} n^{\nu}\right)\right. \\
& \left.\times\left[-k q\left(p^{2} q_{0}-q^{2} p_{0}-2 \mathbf{p} \cdot \mathbf{q}\left(p_{0}-q_{0}\right)\right)+4 k_{0}(p q) p_{0} q_{0}+q^{2} \mathbf{p}^{2} q_{0}+p^{2} \mathbf{q}^{2} p_{0}-\left(p^{2} q_{0}+q^{2} p_{0}\right) \mathbf{p} \cdot \mathbf{q}\right]+(p \leftrightarrow q)\right\} \\
& \left.+n^{\mu} n^{\nu} 4 k^{2}(p q) p_{0} q_{0}\right]+\xi_{C} \frac{N}{2} g^{2} \int d p \frac{1}{p^{2} q^{2} \mathbf{p}^{4} \mathbf{q}^{4}}\left[g^{\mu \nu} k^{2}\left\{k^{2}\left(p^{2} \mathbf{q}^{4}+q^{2} \mathbf{p}^{4}\right)-p^{2} q^{2}\left(\mathbf{p}^{4}+\mathbf{q}^{4}\right)\right\}\right. \\
& +\left\{p^{\mu} p^{\nu}\left[q^{2} \mathbf{p}^{2}\left(2 k q\left(k_{0} p_{0}\right)+(k q)^{2}-k^{2} \mathbf{p}^{2}\right)+p^{2} \mathbf{p}^{2}\left(-2 k q\left(k_{0} q_{0}\right)+(k q)^{2}-k^{2} \mathbf{q}^{2}\right)+p^{2} q^{2}\left(\mathbf{q}^{4}+\mathbf{p}^{2}\left(2 k q+\mathbf{p}^{2}\right)\right)\right]\right. \\
& +(p \leftrightarrow q)\}+\left(p^{\mu} q^{\nu}+q^{\mu} p^{\nu}\right)\left\{p^{2} \mathbf{q}^{2}\left[\left(k_{0} q_{0}\right)\left(q^{2}-p^{2}\right)-(k p)(k q)-k^{2} \mathbf{q}^{2}\right]+p^{2} q^{2} \mathbf{q}^{2}\left[\mathbf{q}^{2}-k q\right]+(p \leftrightarrow q)\right\} \\
& \left.+\left\{\left(n^{\mu} p^{\nu}+p^{\mu} n^{\nu}\right) k q\left[k^{2}\left(q^{2} \mathbf{p}^{2} p_{0}-p^{2} \mathbf{q}^{2} q_{0}\right)-p^{2} q^{2}\left(\mathbf{p}^{2} p_{0}-\mathbf{q}^{2} q_{0}\right)\right]+(p \leftrightarrow q)\right\}\right]+\xi_{C}^{2} \frac{N}{2} g^{2} \int d p \frac{1}{\mathbf{p}^{4} \mathbf{q}^{4}} \\
& \times\left[\left\{-(k q)^{2} p^{\mu} p^{\nu}+(p \leftrightarrow q)\right\}+(k p)(k q)\left(p^{\mu} q^{\nu}+q^{\mu} p^{\nu}\right)\right]
\end{aligned}
$$

\section{APPENDIX B: TEMPORAL AXIAL GAUGE}

\section{Pinch contribution}

Note that the gauge parameter $\xi_{A}$ has a dimension mass ${ }^{-2}$.

(i) The pinch contribution from the vertices of the first kind:

$$
\Pi_{P(\mathrm{TAG})}^{\mu \nu\left(V_{1}\right)}=\frac{N}{2} g^{2} k^{2} d^{\mu \nu} \int d p\left(\frac{-1}{p^{2} p_{0}^{2}}+\frac{-1}{q^{2} q_{0}^{2}}\right)+\xi_{A} \frac{N}{2} g^{2} k^{2} d^{\mu \nu} \int d p\left(\frac{-1}{p_{0}^{2}}+\frac{-1}{q_{0}^{2}}\right) .
$$

(ii) The contribution of the vertices of the second kind:

$$
\begin{aligned}
\Pi_{P(\mathrm{TAG})}^{\mu \nu\left(V_{2}\right)}= & N g^{2} k^{2} d^{\mu \nu} \int d p \frac{1}{p^{2} q^{2}}\left\{\frac{p^{2}}{p_{0}^{2}}+\frac{q^{2}}{q_{0}^{2}}-\frac{2 \mathbf{k} \cdot \mathbf{p}}{p_{0}^{2}}-\frac{2 \mathbf{k} \cdot \mathbf{q}}{q_{0}^{2}}\right\}+N g^{2} k^{2} d^{\mu \alpha} d^{\nu \beta} \int d p \frac{1}{p^{2} q^{2} p_{0}^{2} q_{0}^{2}}\left\{p_{\alpha} p_{\beta}\left[\mathbf{k}^{2}-p_{0}^{2}-q_{0}^{2}\right]\right. \\
& \left.+n_{\alpha} n_{\beta} p_{0} q_{0}\left(k^{2}+2 p q\right)+\left(p_{\alpha} n_{\beta}+n_{\alpha} p_{\beta}\right)\left[\frac{1}{2}\left(p_{0} p^{2}-q_{0} q^{2}\right)-p_{0}\left(\mathbf{q}^{2}+2 \mathbf{p} \cdot \mathbf{q}\right)+q_{0}\left(\mathbf{p}^{2}+2 \mathbf{p} \cdot \mathbf{q}\right)\right]\right\} \\
& +\xi_{A} N g^{2}\left[k^{2} d^{\mu \nu} \int d p\left\{-k^{2}\left(\frac{1}{q^{2} p_{0}^{2}}+\frac{1}{p^{2} q_{0}^{2}}\right)+\frac{1}{p_{0}^{2}}+\frac{1}{q_{0}^{2}}\right\}+k^{2} d^{\mu \alpha} d^{\nu \beta} \int d p \frac{1}{p_{0}^{2} q_{0}^{2}}\left\{p_{\alpha} p_{\beta}\left[-k^{2}\left(\frac{1}{p^{2}}+\frac{1}{q^{2}}\right)\right]\right.\right. \\
& \left.\left.+\left(p_{\alpha} n_{\beta}+n_{\alpha} p_{\beta}\right)\left[\frac{1}{2}\left(p_{0}-q_{0}\right)+p_{0} \frac{q^{2}+2 p q}{p^{2}}-q_{0} \frac{p^{2}+2 p q}{q^{2}}\right]\right\}\right]+\xi_{A}^{2} N g^{2} k^{4} d^{\mu \alpha} d^{\nu \beta} \int d p \frac{-p_{\alpha} p_{\beta}}{p_{0}^{2} q_{0}^{2}} .
\end{aligned}
$$

(iii) The pinch contribution from the box diagrams:

$$
\begin{aligned}
\Pi_{P(\mathrm{TAG})}^{\mu \nu(\mathrm{box})}= & \frac{N}{2} g^{2} k^{4} d^{\mu \nu} \int d p \frac{1}{p^{2} q^{2}}\left(\frac{1}{p_{0}^{2}}+\frac{1}{q_{0}^{2}}\right)+\frac{N}{2} g^{2} k^{4} d^{\mu \alpha} d^{\nu \beta} \int d p \frac{p_{\alpha} p_{\beta}+\left(p_{\alpha} n_{\beta}+n_{\alpha} p_{\beta}\right)\left(q_{0}-p_{0}\right)-2 n_{\alpha} n_{\beta} p_{0} q_{0}}{p^{2} q^{2} p_{0}^{2} q_{0}^{2}} \\
& +\xi_{A} \frac{N}{2} g^{2} k^{4}\left[d^{\mu \nu} \int d p\left(\frac{1}{q^{2} p_{0}^{2}}+\frac{1}{p^{2} q_{0}^{2}}\right)+d^{\mu \alpha} d^{\nu \beta} \int d p\left\{\frac{p_{\alpha} p_{\beta}}{p_{0}^{2} q_{0}^{2}}\left(\frac{1}{p^{2}}+\frac{1}{q^{2}}\right)+\frac{p_{\alpha} n_{\beta}+n_{\alpha} p_{\beta}}{p_{0}^{2} q_{0}^{2}}\left(\frac{q_{0}}{q^{2}}-\frac{p_{0}}{p^{2}}\right)\right\}\right] \\
& +\xi_{A}^{2} \frac{N}{2} g^{2} k^{4} d^{\mu \alpha} d^{\nu \beta} \int d p \frac{p_{\alpha} p_{\beta}}{p_{0}^{2} q_{0}^{2}} .
\end{aligned}
$$

\section{Expression of $\Pi_{P(\mathrm{TAG})}^{\mu \nu}$}

The pinch contribution to the gluon self-energy in the TAG is rewritten in terms of symmetric tensors $g_{\mu \nu}, p_{\mu} p_{\nu}, q_{\mu} q_{\nu}$, $\left(p_{\mu} q_{\nu}+q_{\mu} p_{\nu}\right),\left(n_{\mu} p_{\nu}+p_{\mu} n_{\nu}\right),\left(n_{\mu} q_{\nu}+q_{\mu} n_{\nu}\right)$, and $n_{\mu} n_{\nu}$ : 


$$
\begin{aligned}
\Pi_{P(\mathrm{TAG})}^{\mu \nu}(k)= & \frac{N}{2} g^{2} \int d p \frac{1}{p^{2} q^{2}}\left[g^{\mu \nu}\left\{\frac{k^{2}\left(k^{2}+2 p^{2}-q^{2}-4 \mathbf{k} \cdot \mathbf{p}\right)}{p_{0}^{2}}+(p \leftrightarrow q)\right\}+\left\{p ^ { \mu } p ^ { \nu } \left[-3-\frac{(\mathbf{p} \cdot \mathbf{q})^{2}}{p_{0}^{2} q_{0}^{2}}+\frac{2 \mathbf{p} \cdot \mathbf{q}}{p_{0}^{2} q_{0}^{2}}\left(q^{2}-2 q_{0}^{2}\right)\right.\right.\right. \\
& \left.\left.-\frac{q^{4}}{p_{0}^{2} q_{0}^{2}}+\frac{p^{2}}{p_{0}^{2}}-\frac{q^{2}}{q_{0}^{2}}\right]+(p \leftrightarrow q)\right\}+\left(p^{\mu} q^{\nu}+q^{\mu} p^{\nu}\right)\left[-5+\frac{(\mathbf{p} \cdot \mathbf{q})^{2}}{p_{0}^{2} q_{0}^{2}}-(\mathbf{p} \cdot \mathbf{q})\left(\frac{p^{2}+q^{2}}{p_{0}^{2} q_{0}^{2}}+\frac{2}{p_{0}^{2}}+\frac{2}{q_{0}^{2}}\right)+\frac{p^{2} q^{2}}{p_{0}^{2} q_{0}^{2}}\right] \\
& +\left\{\left(n^{\mu} p^{\nu}+p^{\mu} n^{\nu}\right) \frac{1}{p_{0}^{2} q_{0}^{2}}\left[-k q\left(-p^{2} q_{0}+q^{2} p_{0}-2 \mathbf{p} \cdot \mathbf{q}\left(p_{0}-q_{0}\right)\right)+4 k_{0} p_{0} q_{0}(p q)\right]+(p \leftrightarrow q)\right\} \\
& \left.+n^{\mu} n^{\nu} \frac{4 p_{0} q_{0} k^{2}(p q)}{p_{0}^{2} q_{0}^{2}}\right]+\xi_{A} \frac{N}{2} g^{2} \int d p\left[g^{\mu \nu}\left\{\left(-k^{4} \frac{1}{q^{2} p_{0}^{2}}+k^{2} \frac{1}{p_{0}^{2}}\right)+(p \leftrightarrow q)\right\}\right. \\
& +\left\{p^{\mu} p^{\nu}\left[k^{2}\left(\frac{1}{p^{2} q_{0}^{2}}+\frac{1}{q^{2} p_{0}^{2}}\right)-\frac{(k q)^{2}}{p_{0}^{2} q_{0}^{2}}\left(\frac{1}{p^{2}}+\frac{1}{q^{2}}\right)+\frac{2 k_{0}(k q)}{p_{0}^{2} q_{0}^{2}}\left(\frac{q_{0}}{q^{2}}-\frac{p_{0}}{p^{2}}\right)-\frac{3}{p_{0}^{2}}-\frac{1}{q_{0}^{2}}\right]+(p \leftrightarrow q)\right\} \\
& +\left(p^{\mu} q^{\nu}+q^{\mu} p^{\nu}\right)\left[\left(\frac{k^{2} p_{0}^{2}+(k p)(k q)-k_{0} p_{0}\left(p^{2}-q^{2}\right)}{p^{2} p_{0}^{2} q_{0}^{2}}-\frac{2}{p_{0}^{2}}\right)+(p \leftrightarrow q)\right] \\
& \left.+\left\{\left(n^{\mu} p^{\nu}+p^{\mu} n^{\nu}\right) \frac{k q}{p_{0}^{2} q_{0}^{2}}\left[-k^{2}\left(\frac{p_{0}}{p^{2}}-\frac{q_{0}}{q^{2}}\right)-q_{0}+p_{0}\right]+(p \leftrightarrow q)\right\}\right] \\
& +\xi_{A}^{2} \frac{N}{2} g^{2} \int d p \frac{1}{p_{0}^{2} q_{0}^{2}}\left[\left\{-(k q)^{2} p^{\mu} p^{\nu}+(p \leftrightarrow q)\right\}+(k p)(k q)\left(p^{\mu} q^{\nu}+q^{\mu} p^{\nu}\right)\right] .
\end{aligned}
$$

\section{APPENDIX C: THERMAL ONE-LOOP INTEGRALS}

We list the thermal one-loop integrals in the static limit $k_{0}=0$ which appear in Sec. V. The expressions are in the imaginary time formalism and thus

$$
\int d p=\int \frac{d^{3} p}{(2 \pi)^{3}} T \sum_{n},
$$

where the summation goes over $p_{0}=2 \pi i n T$. We only give the matter part. Because of the constraint $k+p+q=0$ we have

$$
\int d p f(p, q)=\int d p f(q, p) .
$$

It is understood that in the right-hand side (RHS) of the expressions below, $p \equiv|\mathbf{p}|, \kappa \equiv|\mathbf{k}|$, and $n(p)=1 /[\exp (p / T)-1]$ :

$$
\begin{gathered}
\mathbf{k}^{2} \int d p \frac{\mathbf{k}^{2}+4 \mathbf{k} \cdot \mathbf{p}}{p^{2} q^{2} p_{0}^{2}}=\frac{1}{4 \pi^{2}} \int_{0}^{\infty} d p p n(p)\left(-\frac{\kappa^{3}}{p^{3}}\right) \ln \left|\frac{2 p+\kappa}{2 p-\kappa}\right|, \\
\mathbf{k}^{2} \int d p \frac{1}{q^{2} p_{0}^{2}}=\frac{1}{4 \pi^{2}} \int_{0}^{\infty} d p p n(p)\left(-2 \frac{\kappa^{2}}{p^{2}}\right), \\
\mathbf{k}^{2} \int d p \frac{1}{p^{2} p_{0}^{2}}=\frac{1}{4 \pi^{2}} \int_{0}^{\infty} d p p n(p)\left(-2 \frac{\kappa^{2}}{p^{2}}\right), \\
\mathbf{k}^{4} \int d p\left[\mathbf{p}^{2}-\frac{(\mathbf{k} \cdot \mathbf{p})^{2}}{\mathbf{k}^{2}}\right] \frac{1}{p^{2} q^{2} p_{0}^{2} q_{0}^{2}}=\frac{1}{4 \pi^{2}} \int_{0}^{\infty} d p p n(p)\left\{\frac{\kappa^{4}}{p^{4}}+\frac{\kappa^{3}\left(4 p^{2}-\kappa^{2}\right)}{4 p^{5}} \ln \left|\frac{2 p+\kappa}{2 p-\kappa}\right|\right\}, \\
\mathbf{k}^{2} \int d p\left[\mathbf{p}^{2}-\frac{(\mathbf{k} \cdot \mathbf{p})^{2}}{\mathbf{k}^{2}}\right] \frac{1}{p^{2} q^{2} p_{0}^{2}}=\frac{1}{4 \pi^{2}} \int_{0}^{\infty} d p p n(p)\left\{\frac{\kappa^{2}}{p^{2}}+\frac{\kappa\left(4 p^{2}-\kappa^{2}\right)}{4 p^{3}} \ln \left|\frac{2 p+\kappa}{2 p-\kappa}\right|\right\} .
\end{gathered}
$$


[1] J. M. Cornwall, in Proceedings of the French-American Seminar on Theoretical Aspects of Quantum Chromodynamics, Marseille, France, 1981, edited by J. W. Dash (Centre de Physique Théorique, Marseille, 1982); Phys. Rev. D 26, 1453 (1982).

[2] J. M. Cornwall and J. Papavassiliou, Phys. Rev. D 40, 3474 (1989).

[3] J. Papavassiliou, Phys. Rev. D 47, 4728 (1993).

[4] J. Papavassiliou, Phys. Rev. D 41, 3179 (1990); G. Degrassi and A. Sirlin, ibid. 46, 3104 (1992); J. Papavassiliou and K. Philippides, ibid. 48, 4255 (1993); 52, 2355 (1995); G. Degrassi, B. Kniehl, and A. Sirlin, ibid. 48, R3963 (1993); J. Papavassiliou and C. Parrinello, ibid. 50, 3059 (1994); J. Papavassiliou, and A. Sirlin, ibid. 50, 5951 (1994); J. Papavassiliou, ibid. 50, 5958 (1994); K. Hagiwara, D. Haidt, C. S. Kim, and S. Matsumoto, Z. Phys. C 64, 559 (1994); J. Papavassiliou, K. Philippides, and M. Schaden, Phys. Rev. D 51, 6364 (1995); J. Papavassiliou and A. Pilaftsis, Phys. Rev. Lett. 75, 3060 (1995); Phys. Rev. D 53, 2128 (1996); J. Papavassiliou, K. Philippides, and K. Sasaki, ibid. 53, 3942 (1996); K. Philippides and A. Sirlin, Phys. Lett. B 367, 377 (1996).

[5] G. Alexanian and V. P. Nair, Phys. Lett. B 352, 435 (1995).

[6] K. Sasaki, Phys. Lett. B 369, 117 (1996).

[7] K. Sasaki, Nucl. Phys. B472, 271 (1996).

[8] N. J. Watson, Phys. Lett. B 349, 155 (1995).

[9] A. Denner, S. Dittmaier, and G. Weiglein, Phys. Lett. B 333, 420 (1994); Nucl. Phys. B440, 95 (1995); S. Hashimoto, J. Kodaira, K. Sasaki, and Y. Yasui, Phys. Rev. D 50, 7066 (1994); E. de Rafael and N. J. Watson (unpublished).

[10] J. Papavassiliou, Phys. Rev. D 51, 856 (1995).

[11] G. Leibbrandt, Noncovariant Gauges (World Scientific, Singapore, 1994); see also Physical and Nonstandard Gauges, edited by P. Gaigg, W. Kummer, and M. Schweda, Lecture Notes in Physics Vol. 361 (Springer-Verlag, Berlin, 1990); A. Bassetto, G. Nardelli, and R. Soldati, Yang-Mills Theories in Algebraic Non-Covariant Gauges (World Scientific, Singapore, 1991).
[12] K. Kajantie and J. Kapusta, Ann. Phys. (N.Y.) 160, 477 (1985).

[13] U. Heinz, K. Kajantie, and T. Toimela, Phys. Lett. B 183, 96 (1987); Ann. Phys. (N.Y.) 176, 218 (1987).

[14] E. Braaten and R. D. Pisarski, Phys. Rev. Lett. 64, 1338 (1990); Nucl. Phys. B337, 569 (1990); B339, 310 (1990); Phys. Rev. D 42, 2156 (1990).

[15] K. Kajantie, in Physical and Nonstandard Gauges, edited by P. Gaigg, W. Kummer, and M. Schweda, Lecture Notes in Physics Vol. 361 (Springer-Verlag, Berlin, 1990), p. 263.

[16] G. Leibbrandt, Rev. Mod. Phys. 59, 1067 (1987).

[17] W. Kummer, Acta Phys. Austriaca 41, 315 (1975); J. Frenkel and J. C. Taylor, Nucl. Phys. B109, 439 (1976).

[18] S. Mandelstam, Nucl. Phys. B213, 149 (1983); G. Leibbrandt, Phys. Rev. D 29, 1699 (1984); Nucl. Phys. B310, 405 (1988); G. Leibbrandt and M. Staley, ibid. B428, 469 (1994).

[19] P. V. Landshoff, Phys. Lett. 169B, 69 (1986).

[20] A. Ali, J. Bernstein, and A. Zepeda, Phys. Rev. D 12, 503 (1975).

[21] V. P. Silin, Sov. Phys. JETP 11, 1136 (1960); O. K. Kalashnikov and V. V. Klimov, Sov. J. Nucl. Phys. 31, 699 (1980); V. V. Klimov ibid. 33, 934 (1981); Sov. Phys. JETP 55, 199 (1982); H. A. Weldon, Phys. Rev. D 26, 1394 (1982).

[22] D. M. Capper and G. Leibbrandt, Phys. Rev. D 25, 1002 (1982); 25, 1009 (1982).

[23] N. P. Landsman, Phys. Lett. B 232, 240 (1989); M. A. van Eijck, Can. J. Phys. 71, 237 (1993); M. A. van Eijck, C. R. Stephens, and Ch. G. van Weert, Mod. Phys. Lett. A 9, 309 (1994).

[24] J. Antikainen, M. Chaichian, N. R. Pantoja, and J. J. Salazar, Phys. Lett. B 242, 412 (1990).

[25] P. Elmfors and R. Kobes, Phys. Rev. D 51, 774 (1995).

[26] K. Sasaki, "Gauge-independent resummed gluon self-energy in hot QCD,', report (in preparation).

[27] J. Papavassiliou and A. Pilaftsis, Phys. Rev. D 54, 5315 (1996). 